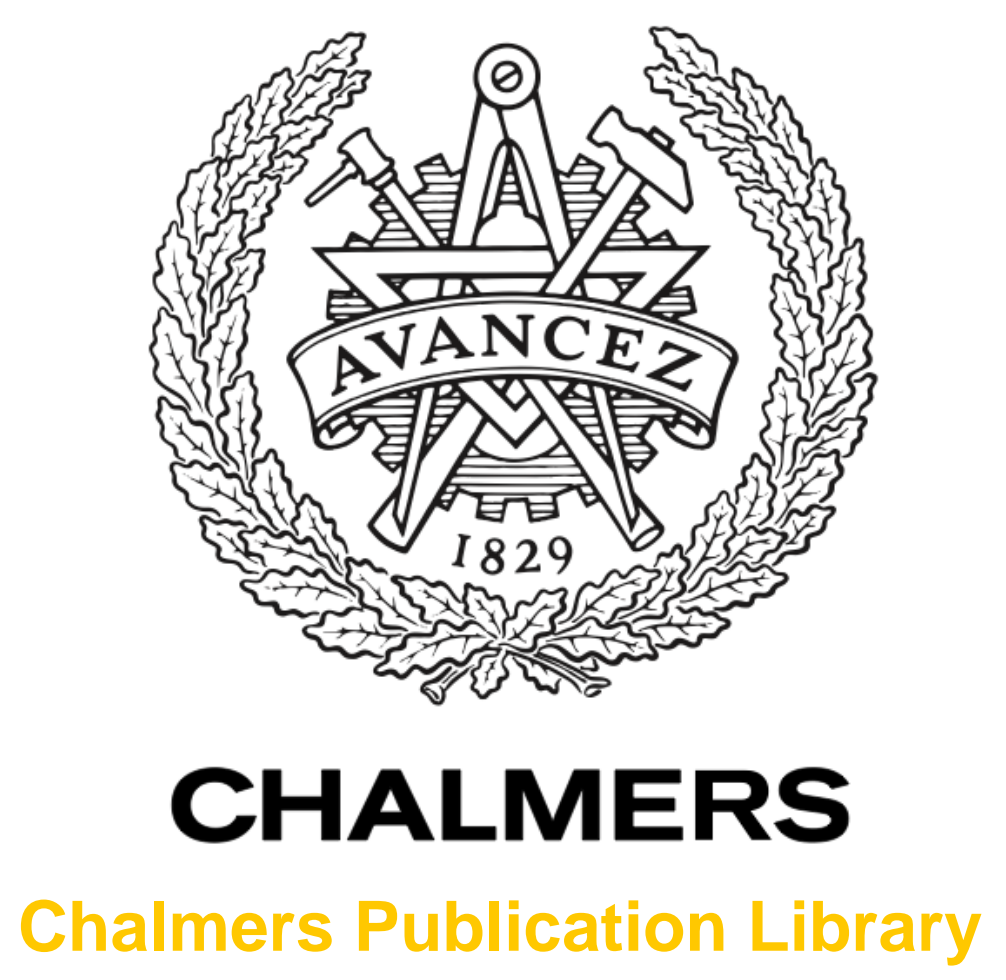

\title{
Dispersion free wave splittings for structural elements
}

This document has been downloaded from Chalmers Publication Library (CPL). It is the author's version of a work that was accepted for publication in:

Computers \& structures (ISSN: 0045-7949)

Citation for the published paper:

Johansson, M. ; Folkow, P. ; Olsson, P. (2006) "Dispersion free wave splittings for structural elements". Computers \& structures, vol. 84 pp. 514-27.

http://dx.doi.org/10.1016/j.compstruc.2005.09.006

Downloaded from: http://publications.lib.chalmers.se/publication/24391

Notice: Changes introduced as a result of publishing processes such as copy-editing and formatting may not be reflected in this document. For a definitive version of this work, please refer to the published source. Please note that access to the published version might require a subscription.

Chalmers Publication Library (CPL) offers the possibility of retrieving research publications produced at Chalmers University of Technology. It covers all types of publications: articles, dissertations, licentiate theses, masters theses, conference papers, reports etc. Since 2006 it is the official tool for Chalmers official publication statistics. To ensure that Chalmers research results are disseminated as widely as possible, an Open Access Policy has been adopted.

The CPL service is administrated and maintained by Chalmers Library. 


\title{
Dispersion Free Wave Splittings for Structural Elements
}

\author{
M.Johansson, P.D. Folkow* ${ }^{*}$ P. Olsson. \\ Department of Applied mechanics, Chalmers University of Technology, \\ SE-412 96 Göteborg, Sweden.
}

\begin{abstract}
Wave splittings are derived for three types of structural elements: membranes, Timoshenko beams, and Mindlin plates. The Timoshenko beam equation and the Mindlin plate equation are inherently dispersive, as is each Fourier component of the membrane equation in an angular decomposition of the field. The distinctive feature of the wave splittings derived in the present paper is that, in homogeneous regions, they transform the dispersive wave equations into simple one-way wave equations without dispersion. Such splittings have uses both for radial scattering problems in the 2D cases and for scattering problems in dispersive media. As an example of how the splittings may be applied, a direct scattering problem is solved for a membrane with radially varying density. The imbedding method is utilised, and agreement is obtained with an FE simulation.
\end{abstract}

Key words: wave splitting, time domain methods, Green's operator, imbedding, membrane, Timoshenko beam, Mindlin plate

\section{Introduction}

During the last decades, time domain methods such as invariant imbedding, Green's function techniques and propagator methods, have successfully been applied to a number of scattering and wave propagation problems. A survey of these methods is contained in [1]. Both direct and inverse scattering problems have been considered, as well as problems concerning, i.e., wave guides

* Tel: +46 31 7721521, Fax: +46 317723827 .

Email addresses: martin.johansson@me.chalmers.se (M.Johansson), peter.folkow@me.chalmers.se (P.D. Folkow), peter.olsson@me.chalmers.se (P. Olsson).

Preprint submitted to Computers and Structures 2 January 2004 
and slender structural elements. Much of the work has been concerned with electromagnetics, but acoustics and structural mechanics have also benefited from the time domain approaches, see e.g. [2-6]. One basic building block in such time domain methods is the wave splitting; i.e., a transformation that achieves a decomposition of the wave fields into one-way waves. A number of such splittings have been derived, with different properties and applications. For some purposes, the decomposition need only diagonalise the principal part of the operator matrix of the relevant differential equation. Often it is advantageous to have a transformation that exactly splits the PDE into one-way wave equations in the homogeneous region. If the original wave equation is dispersive, the split wave equations in general are so too even in the homogeneous regions. In regions where the original wave equations model inhomogeneities, the one-way wave equations couple, and the coupling may often be utilised for the reconstruction of the inhomogeneities. We refer to $[1,7]$ for how this may be done numerically.

Much of the work on wave splitting has been concerned with $1 \mathrm{D}$ wave propagation, or situations which may naturally be reduced to 1D propagation. Weston and collaborators have, however, done much to extend the wave splittings to more general situations, see e.g. [8,9]. Moreover, Karlsson and Kristensson [10] have treated a $3 \mathrm{D}$ radially symmetric scattering problem, and Kreider $[11,12]$ has solved an inverse $2 \mathrm{D}$ radially symmetric scattering problem.

In the present paper we consider 1D and 2D wave propagation in some structural elements. In all 2D cases considered, a distinguished radial direction allows us to reduce the problem to $1 \mathrm{D}$ propagation. However, common to both the $1 \mathrm{D}$ and $2 \mathrm{D}$ wave propagation for the structural elements considered is that the wave equations exhibit dispersion, either directly geometrical or indirectly geometrical. By directly geometrical dispersion we here denote dispersion due to space dependent coefficients of the wave equations, as in the case of radial wave equations. By indirectly geometrical dispersion we denote such dispersion that arises from the modeling of the structural elements as lower dimensional objects, reducing the wave equation from $3 \mathrm{D}$ to $1 \mathrm{D}$ or $2 \mathrm{D}$ in space variables.

Preliminary considerations on the radial membrane showed that equations exhibiting directly geometrical dispersion can not be split by means of the standard methods utilised for indirectly geometrically dispersive equations. The method which is presented in this paper, not only achieves an exact wave splitting in homogeneous regions, but also provides some freedom to choose the split wave equations. Here, we may choose dispersion free one-way wave equations in a straightforward way. This method can also be utilised to obtain wave splittings for equations that are not directly geometrically dispersive, such as the Timoshenko beam equation. The same result may also be achieved by extending the standard wave splitting by means of Green's operators, which is demonstrated in Section 2. It should be stressed, though, that this latter 
approach is not an option for the radial cases.

A key feature of the dispersion free wave splittings is that the Green's operators are incorporated into the wave splitting transformation. The computational difficulties involved when wave fields are propagated are thus not eliminated entirely by the dispersion free wave splitting. All information about the dispersion of the physical fields is contained in the wave splitting transformation, which of course must be computed.

In Section 3 we derive the dispersion free wave splitting on the radial membrane. The derivation can be generalised and incorporated into the analysis of the radial Mindlin plate in Section 4. The plate problem is in a sense a combination of the beam and the membrane problems, as it contains both the indirectly geometrical dispersion of the beam, and the directly geometrical dispersion of the radial membrane In Section 5 we apply the wave splitting to an inhomogeneous membrane. The imbedding equations is discussed and implemented numerically for some direct problems. The results are compared to FE solutions. Some concluding remarks are found in Section 6.

Our goal is then to construct wave splittings that not only diagonalise the original wave equations, but also remove both the directly and the indirectly geometrical dispersion.

\section{The Timoshenko Beam}

This section first presents a brief review of the original wave splitting transformation together with new results on how to transform to dispersion free fields. Then follows an alternative method of obtaining dispersion free fields, which has the advantage of being more easily extended to the cases of the membrane and the plate.

The Timoshenko beam equation may be written [13]

$$
\begin{aligned}
& \partial_{x} \gamma=c_{1}^{-2} \partial_{t}^{2} w \\
& \partial_{x}^{2} \psi-f \gamma=c_{2}^{-2} \partial_{t}^{2} \psi \\
& \gamma=\partial_{x} w+\psi
\end{aligned}
$$

where $w(x, t), \psi(x, t)$ and $\gamma(x, t)$ are the mean transverse deflection, the mean rotation and the mean shear angle of the cross section, respectively. Note that in $[13-15] \psi$ is defined in the opposite direction.

The quotient of the shearing and bending stiffnesses $f$, the velocities $c_{1}$ (ef- 
fective shear velocity) and $c_{2}$ (rod velocity) are defined as

$$
f=\sqrt{k^{\prime} G A / I E}, \quad c_{1}=\sqrt{k^{\prime} G / \rho}, \quad c_{2}=\sqrt{E / \rho},
$$

Here, $A$ and $I$ are the area and the moment of inertia of the cross section, while $\rho$ is the density of the beam. $E$ is the modulus of elasticity, $G$ is the shear modulus and $k^{\prime}$ is the shear coefficient.

\subsection{Standard wave splitting}

The original wave splitting for the Timoshenko beam was presented by Olsson and Kristensson [14], with an amendment given by Folkow et al. [15]. It takes its starting point from (1)-(3), written as a spatially first order system according to

$$
\partial_{x} \boldsymbol{w}=\mathcal{D} \boldsymbol{w}, \quad \mathcal{D}=\left(\begin{array}{cccc}
0 & -1 & 1 & 0 \\
0 & 0 & 0 & 1 \\
c_{1}^{-2} \partial_{t}^{2} & 0 & 0 & 0 \\
0 & c_{2}^{-2} \partial_{t}^{2} & f & 0
\end{array}\right), \quad \boldsymbol{w}=\left(\begin{array}{c}
w \\
\psi \\
\gamma \\
\partial_{x} \psi
\end{array}\right)
$$

An operator $\mathcal{P}$ and its formal inverse $\mathcal{P}^{-1}$ are introduced and chosen so as to diagonalise $\mathcal{D}$

$$
\boldsymbol{u}^{\circ}=\mathcal{P} \boldsymbol{w}, \quad \boldsymbol{w}=\mathcal{P}^{-1} \boldsymbol{u}^{\circ}, \quad \mathcal{P} \mathcal{D} \mathcal{P}^{-1}=\operatorname{diag}\left(-\lambda_{1},-\lambda_{2}, \lambda_{1}, \lambda_{2}\right)
$$

where $\boldsymbol{u}^{\circ}=\left(u_{1}^{+}, u_{1}^{-}, u_{2}^{+}, u_{2}^{-}\right)^{T}$ is the vector of the split fields and the $\lambda_{i}$ are eigenoperators of $\mathcal{D}$. The transformation operators are normalised so that $w=u_{1}^{+}+u_{1}^{-}+u_{2}^{+}+u_{2}^{-}$. Hence, the Timoshenko beam equation (4) may be written as dispersive one-way wave equations for the split fields as

$$
\partial_{x} u_{i}^{ \pm} \pm \lambda_{i} u_{i}^{ \pm}=0, \quad \lambda_{i}=\frac{1}{c_{i}} \partial_{t}+F_{i}(\cdot) *
$$

In [14] explicit representations in the time domain for the $\lambda_{i}$, the $F_{i}$ and the elements of $\mathcal{P}$ and $\mathcal{P}^{-1}$ are given.

For homogeneous beams, the left and right moving fields at a position $x$ are related to the left and right moving fields at a position $x^{\prime}$ respectively, through linear operators called wave propagators or Green's operators. These are defined by the following mapping properties [15]

$$
u_{i}^{ \pm}\left(x, t \pm\left(x-x^{\prime}\right) / c_{i}\right)=\mathcal{G}_{i}^{ \pm}\left(x^{\prime}, x\right) u_{i}^{ \pm}\left(x^{\prime}, t\right) .
$$


Note that there are no restrictions on the relative magnitudes of $x$, and $x^{\prime}$ in the Green's operators. Due to the symmetry of the homogeneous beam $\mathcal{G}_{i}^{-}\left(x_{1}, x\right)=\mathcal{G}_{i}^{+}\left(x, x_{1}\right)=\mathcal{G}_{i}\left(x_{1}-x\right)$. The inverse operator may then be written $\mathcal{G}_{i}^{-1}\left(x_{1}-x\right)=\mathcal{G}_{i}\left(x-x_{1}\right)$. So, (7) may be summarised as

$$
u_{i}^{ \pm}\left(x, t \pm\left(x-x^{\prime}\right) / c_{i}\right)=\mathcal{G}_{i}\left( \pm\left(x-x^{\prime}\right)\right) u_{i}^{ \pm}\left(x^{\prime}, t\right) .
$$

The functional form of the Green's operators is obtained by applying Laplace transform techniques to (6) and solving the ordinary differential equations, which gives

$$
\widetilde{u}_{i}^{ \pm}(x, s)=\widetilde{u}_{i}^{ \pm}\left(x^{\prime}, s\right) \mathrm{e}^{\mp \widetilde{\lambda}_{i}(s)\left(x-x^{\prime}\right)}=\widetilde{u}_{i}^{ \pm}\left(x^{\prime}, s\right) \mathrm{e}^{\mp\left(s c_{i}^{-1}+\widetilde{F}_{i}(s)\right)\left(x-x^{\prime}\right)} .
$$

By defining the kernel

$$
\widetilde{G}_{i}\left(x-x^{\prime}, s\right)=\mathrm{e}^{-\widetilde{F}_{i}(s)\left(x-x^{\prime}\right)}-1,
$$

the Green's operator may be represented by $\mathcal{G}_{i}\left(x-x^{\prime}\right)=1+G_{i}\left(x-x^{\prime}, \cdot\right) *$. The kernels $G_{i}$ are Green's functions (in the sense of Krueger and Ochs [16]) and can be obtained from (10) in terms of Volterra equations of the second kind, see [15],

$$
G_{i}\left(x-x^{\prime}, t\right)=-\frac{x-x^{\prime}}{t} \int_{0}^{t} t^{\prime} F_{i}\left(t^{\prime}\right) G_{i}\left(x-x^{\prime}, t-t^{\prime}\right) \mathrm{d} t^{\prime}-\left(x-x^{\prime}\right) F_{i}(t) .
$$

Dispersion free fields $v_{i}^{ \pm}$are obtained by using (9)

$$
\widetilde{v}_{i}^{ \pm}(x, s)=\widetilde{u}_{i}^{ \pm}(x, s) \mathrm{e}^{ \pm \widetilde{F}_{i}(s)\left(x-x^{\prime}\right)}=\widetilde{u}_{i}^{ \pm}\left(x^{\prime}, s\right) \mathrm{e}^{\mp s c_{i}^{-1}\left(x-x^{\prime}\right)},
$$

which gives

$$
v_{i}^{ \pm}(x, t)=u_{i}^{ \pm}\left(x^{\prime}, t \mp\left(x-x^{\prime}\right) / c_{i}\right) .
$$

This means that the fields $v_{i}^{ \pm}$satisfy the dispersion free one-way equations

$$
\partial_{x} v_{i}^{ \pm} \pm \frac{1}{c_{i}} \partial_{t} v_{i}^{ \pm}=0
$$

so that the fields $v_{i}^{+}$and $v_{i}^{-}$propagate in opposite directions at speeds $c_{i}$ without dispersion. The fields $u_{i}^{ \pm}$and $v_{i}^{ \pm}$are seen to be related through

$$
v_{i}^{ \pm}(x, t)=\mathcal{G}_{i}\left(\mp\left(x-x^{\prime}\right)\right) u_{i}^{ \pm}(x, t), \quad u_{i}^{ \pm}(x, t)=\mathcal{G}_{i}\left( \pm\left(x-x^{\prime}\right)\right) v_{i}^{ \pm}(x, t) .
$$

Introducing $\boldsymbol{v}=\left(v_{1}^{+}, v_{2}^{+}, v_{1}^{-}, v_{2}^{-}\right)^{T}$ and $\mathcal{G}=\operatorname{diag}\left(\mathcal{G}_{1}^{-1}, \mathcal{G}_{2}^{-1}, \mathcal{G}_{1}, \mathcal{G}_{2}\right)$, where $\mathcal{G}_{i}=$ $\mathcal{G}_{i}\left(x-x^{\prime}\right)$, the dispersion free wave splitting transformation become

$$
\boldsymbol{v}=\mathcal{G} \mathcal{P} w, \quad \boldsymbol{w}=\mathcal{P}^{-1} \mathcal{G}^{-1} \boldsymbol{v}
$$


The combined transformation may now be used even in nonhomogeneous parts of the beam, where coupling occurs between the fields. All the usual approaches of imbedding, Green's functions and propagators may then be utilised. However, in the present context we leave that aside and move on to another way of approaching the dispersion free wave splitting transformation.

\subsection{Reduction to second order equations}

As in the previous section we will derive the dispersion free wave splitting in two steps, thus obtaining a different factorization of the transform than (15).

Consider again (1)-(3). Eliminating $\psi$ and $\gamma$, one obtains the other familiar form of the Timoshenko beam equation

$$
\left(\partial_{x}^{4}-\left(c_{1}^{-2}+c_{2}^{-2}\right) \partial_{x}^{2} \partial_{t}^{2}+r_{0}^{-2} c_{2}^{-2} \partial_{t}^{2}+c_{1}^{-2} c_{2}^{-2} \partial_{t}^{4}\right) w=0
$$

where $r_{0}=\sqrt{I / E}$ is the radius of gyration. Equation (16) may be factorised and written in terms of the eigenoperators defined in (6),

$$
\left(\partial_{x}^{2}-\lambda_{1}^{2}\right)\left(\partial_{x}^{2}-\lambda_{2}^{2}\right) w=0
$$

The differential operators in (17) could of course be factorised further, giving rise to relations such as (6). However, we refrain from doing so since we later want to treat other cases in an analogous way, where the factorization is less obvious. The operators $\lambda_{i}^{2}$ can be represented by [15]

$$
\lambda_{i}^{2}=\frac{1}{c_{i}^{2}} \partial_{t}^{2} \mp \mathcal{V}, \quad \mathcal{V}=\frac{1}{2 r_{0} c_{2} \tau}\left(1+\frac{2}{\cdot} \mathrm{I}_{2}(\cdot / \tau) *\right),
$$

where $\tau=c_{2} r_{0}\left(c_{1}^{-2}-c_{2}^{-2}\right) / 2$ is a characteristic time and $\mathrm{I}_{2}$ is the modified Bessel function of the first kind and order two. Introduce the fields $u_{1}(x, t)$ and $u_{2}(x, t)$ such that

$$
\left(\partial_{x}^{2}-\lambda_{i}^{2}\right) u_{i}=0, \quad i=1,2, \quad w=u_{1}+u_{2}
$$

In analogy with Mindlin [17] and Vemula and Norris [18] we express the rotation as

$$
\psi_{i}=\mathcal{A}_{i} \partial_{x} u_{1}, \quad i=1,2, \quad \psi=\psi_{1}+\psi_{2},
$$

where the (time domain) operators $\mathcal{A}_{i}$ are independent of the spatial coordinate. Since $u_{i}$ are solutions to (1)-(3) as well, use of (1),(3), (6) and (19) gives

$$
\mathcal{A}_{1}=\mathcal{V} \lambda_{1}^{-2}, \quad \mathcal{A}_{2}=\left(c_{1}^{-2}-c_{2}^{-2}\right) \partial_{t}^{2} \lambda_{2}^{-2}-\mathcal{V} \lambda_{2}^{-2}
$$


Their time domain expressions are given in Appendix A. The physical fields $\boldsymbol{w}=\left(w, \psi, \gamma, \partial_{x} \psi\right)^{T}$ may now be expressed in terms of $\boldsymbol{u}=\left(u_{1}, \partial_{x} u_{1}, u_{2}, \partial_{x} u_{2}\right)^{T}$ using (1)-(3) and (19)-(21),

$$
\begin{aligned}
w & =u_{1}+u_{2}, & \gamma & =\left(1+\mathcal{A}_{1}\right) \partial_{x} u_{1}+\left(1+\mathcal{A}_{2}\right) \partial_{x} u_{2}, \\
\psi & =\mathcal{A}_{1} \partial_{x} u_{1}+\mathcal{A}_{2} \partial_{x} u_{2}, & \partial_{x} \psi & =\mathcal{A}_{1} \lambda_{1}^{2} u_{1}+\mathcal{A}_{2} \lambda_{2}^{2} u_{2} .
\end{aligned}
$$

Denoting this first transform operator by $\mathcal{S}, \boldsymbol{u}=\mathcal{S} \boldsymbol{w}$ and $\boldsymbol{w}=\mathcal{S}^{-1} \boldsymbol{u}$, where $\mathcal{S}^{-1}=\left(\begin{array}{cccc}1 & 0 & 1 & 0 \\ 0 & \mathcal{A}_{1} & 0 & \mathcal{A}_{2} \\ 0 & 1+\mathcal{A}_{1} & 0 & 1+\mathcal{A}_{2} \\ \mathcal{A}_{1} \lambda_{1}^{2} & 0 & \mathcal{A}_{2} \lambda_{2}^{2} & 0\end{array}\right), \mathcal{S}=\left(\begin{array}{cccc}1-\mathcal{N}_{1} & 0 & 0 & -\mathcal{N}_{4} \\ 0 & -1-\mathcal{N}_{2} & 1-\mathcal{N}_{3} & 0 \\ \mathcal{N}_{1} & 0 & 0 & \mathcal{N}_{4} \\ 0 & \mathcal{N}_{2} & \mathcal{N}_{3} & 0\end{array}\right)$

The operators $\mathcal{N}_{i}$ are given in Appendix A. The elements of $\mathcal{S}$ and $\mathcal{S}^{-1}$ may of course be derived using (5), since $u_{i}=u_{i}^{+}+u_{i}^{-}$and $\partial_{x} u_{i}=\lambda_{i}\left(-u_{i}^{+}+u_{i}^{-}\right)$.

The Timoshenko equation is thus reduced to two wave equations with different wave front speeds corresponding to the shear and bending modes respectively. At this point we have not yet performed the wave splitting operation but only decoupled the components of the wave field whose wave fronts propagate with different speeds.

\subsection{Transformation to dispersion free fields}

We now derive the second transformation, which will split the fields $u_{i}$ into dispersion free left and right moving components. The two wave equations in (19) are treated simultaneously by considering the system

$$
\partial_{x} \boldsymbol{u}_{i}=\mathcal{D}_{i} \boldsymbol{u}_{i}, \quad \mathcal{D}_{i}=\left(\begin{array}{cc}
0 & 1 \\
\lambda_{i}^{2} & 0
\end{array}\right), \quad \boldsymbol{u}_{i}=\left(\begin{array}{c}
u_{i} \\
\partial_{x} u_{i}
\end{array}\right)
$$

Introduce the operator $\mathcal{B}_{i}$, its inverse $\mathcal{B}_{i}^{-1}$ and dispersion free fields $\boldsymbol{v}_{i}=\left(v_{i}^{+}, v_{i}^{-}\right)^{T}$, such that

$$
\boldsymbol{v}_{i}=\mathcal{B}_{i} \boldsymbol{u}_{i}, \quad \boldsymbol{u}_{i}=\mathcal{B}_{i}^{-1} \boldsymbol{v}_{i}, \quad \partial_{x} \boldsymbol{v}_{i}=\boldsymbol{\Lambda}_{i} \boldsymbol{v}_{i}
$$


where $\boldsymbol{\Lambda}_{i}=c_{i}^{-1} \operatorname{diag}\left\{-\partial_{t}, \partial_{t}\right\}$. Now, as $v_{i}^{+}$and $v_{i}^{-}$satisfy first order one-way wave equations they may be expressed as

$$
v_{i}^{ \pm}(x, t)=v_{i}^{ \pm}\left(x^{\prime}, t \mp\left(x-x^{\prime}\right) / c_{i}\right),
$$

where $x^{\prime}$ is some reference position. Using $u_{i}=\mathcal{B}_{i, 11}^{-1} v_{i}^{+}+\mathcal{B}_{i, 12}^{-1} v_{i}^{-}$in (23) and adopting Laplace transform techniques, the ordinary differential equations are solved as

$$
\begin{aligned}
& \widetilde{\mathcal{B}}_{i, 11}^{-1}=a_{1}(s) \mathrm{e}^{\left(s c_{i}^{-1}+\widetilde{\lambda}_{i}(s)\right)\left(x-x^{\prime}\right)}+b_{1}(s) \mathrm{e}^{\left(s c_{i}^{-1}-\widetilde{\lambda}_{i}(s)\right)\left(x-x^{\prime}\right)}, \\
& \widetilde{\mathcal{B}}_{i, 12}^{-1}=a_{2}(s) \mathrm{e}^{-\left(s c_{i}^{-1}+\widetilde{\lambda}_{i}(s)\right)\left(x-x^{\prime}\right)}+b_{2}(s) \mathrm{e}^{-\left(s c_{i}^{-1}-\widetilde{\lambda}_{i}(s)\right)\left(x-x^{\prime}\right)} .
\end{aligned}
$$

As $v_{i}^{+}$propagates in the positive $x$-direction while $v_{i}^{-}$propagate in the negative $x$-direction, we should have $a_{1}(s)=a_{2}(s)=0$. If we set $b_{1}(s)=b_{2}(s)=1$, the same normalization is used as in Section 2.1. It is straightforward to calculate $\widetilde{\mathcal{B}}_{i, 21}^{-1}$ and $\widetilde{\mathcal{B}}_{i, 22}^{-1}$ using $(6)$. Then,

$$
\begin{aligned}
\widetilde{\mathcal{B}}_{i}^{-1} & =\left(\begin{array}{cc}
\mathrm{e}^{-\widetilde{F}_{i}(s)\left(x-x^{\prime}\right)} & \mathrm{e}^{\widetilde{F}_{i}(s)\left(x-x^{\prime}\right)} \\
-\widetilde{\lambda}_{i}(s) \mathrm{e}^{-\widetilde{F}_{i}(s)\left(x-x^{\prime}\right)} & \widetilde{\lambda}_{i}(s) \mathrm{e}^{\widetilde{F}_{i}(s)\left(x-x^{\prime}\right)}
\end{array}\right), \\
\widetilde{\mathcal{B}}_{i} & =\frac{1}{2}\left(\begin{array}{cc}
\mathrm{e}^{\widetilde{F}_{i}(s)\left(x-x^{\prime}\right)} & -\widetilde{\lambda}_{i}^{-1}(s) \mathrm{e}^{\widetilde{F}_{i}(s)\left(x-x^{\prime}\right)} \\
\mathrm{e}^{-\widetilde{F}_{i}(s)\left(x-x^{\prime}\right)} & \widetilde{\lambda}_{i}^{-1}(s) \mathrm{e}^{-\widetilde{F}_{i}(s)\left(x-x^{\prime}\right)}
\end{array}\right) .
\end{aligned}
$$

In the time domain, we have

$$
\mathcal{B}_{i}^{-1}=\left(\begin{array}{cc}
\mathcal{G}_{i}^{+} & \mathcal{G}_{i}^{-} \\
-\lambda_{i} \mathcal{G}_{i}^{+} & \lambda_{i} \mathcal{G}_{i}^{-}
\end{array}\right), \quad \mathcal{B}_{i}=\frac{1}{2}\left(\begin{array}{cc}
\mathcal{G}_{i}^{-} & -\lambda_{i}^{-1} \mathcal{G}_{i}^{-} \\
\mathcal{G}_{i}^{+} & \lambda_{i}^{-1} \mathcal{G}_{i}^{+}
\end{array}\right)
$$

where $\mathcal{G}_{i}^{+}=\mathcal{G}_{i}\left(x-x^{\prime}\right)$ and $\mathcal{G}_{i}^{-}=\mathcal{G}_{i}\left(x^{\prime}-x\right)$. This is in line with the results in (14) using $u_{i}=u_{i}^{+}+u_{i}^{-}$and $\partial_{x} u_{i}=\lambda_{i}\left(-u_{i}^{+}+u_{i}^{-}\right)$. The operators $\lambda_{i}^{-1}$ are found in Appendix A. By defining $\boldsymbol{v}=\left(\boldsymbol{v}_{1}, \boldsymbol{v}_{2}\right)^{T}$ and $\mathcal{B}=\operatorname{diag}\left(\mathcal{B}_{1}, \mathcal{B}_{2}\right)$ we obtain

$$
\boldsymbol{v}=\mathcal{B S} \boldsymbol{w}, \quad \boldsymbol{w}=\mathcal{S}^{-1} \mathcal{B}^{-1} \boldsymbol{v}
$$

The connection between (15) and (28) is readily seen if $\boldsymbol{\mathcal { B }}_{i}^{-1}$ and $\boldsymbol{\mathcal { B }}_{i}$ are factorised as

$$
\mathcal{B}_{i}^{-1}=\left(\begin{array}{cc}
\mathcal{I} & \mathcal{I} \\
-\lambda_{i} & \lambda_{i}
\end{array}\right)\left(\begin{array}{cc}
\mathcal{G}_{i} & 0 \\
0 & \mathcal{G}_{i}^{-1}
\end{array}\right), \quad \mathcal{B}_{i}=\left(\begin{array}{cc}
\mathcal{G}_{i}^{-1} & 0 \\
0 & \mathcal{G}_{i}
\end{array}\right) \frac{1}{2}\left(\begin{array}{cc}
\mathcal{I}-\lambda_{i}^{-1} \\
\mathcal{I} & \lambda_{i}^{-1}
\end{array}\right)
$$

Hence, the Green's operator matrices carry the spatial dependences that deal with the dispersion, while the eigenoperator matrices are involved in the actual 
wave splitting. This is the reason why the original wave splitting transformation for the Timoshenko beam is found by means of a diagonalization, as the beam is left-right isotropic. A membrane or a plate with rotational symmetry lack the corresponding inward-outward symmetry.

\section{The Membrane}

In this section the dispersion free transformation is derived for radial waves in a homogeneous membrane. The objective is to obtain radially in- and outgoing waves that are uncoupled and propagate without dispersion. Since we have cylindrical geometry we will encounter geometrical dispersion. So, the transformation is performed in line with the methods presented in Section 2.3

The wave equation for the membrane is

$$
\nabla^{2} w(r, \theta, t)-c^{-2} \partial_{t}^{2} w(r, \theta, t)=0
$$

where $w(r, \theta, t)$ is the deflection, $\nabla^{2}$ is the two-dimensional Laplace operator, $c=\sqrt{T / \rho}$ is the wave speed and $\rho, T$ are mass per unit area and tension per unit length respectively. In order to make comparisons to the plate in Section 4 more apparent, the membrane equation is written

$$
\left(\nabla^{2}-\lambda^{2}\right) w=0, \quad \lambda^{2}=c^{-2} \partial_{t}^{2} .
$$

As the aim is to find a transformation for radial waves, $w$ is expanded according to

$$
w(r, \theta, t)=w_{0}^{e}(r, t)+\sum_{m=1}^{\infty}\left(w_{m}^{e}(r, t) \cos (m \theta)+w_{m}^{o}(r, t) \sin (m \theta)\right)
$$

For each Fourier component of the physical variables we may write (30) as

$$
\partial_{r} \boldsymbol{w}_{m}=\mathcal{D}_{m} \boldsymbol{w}_{m}, \quad \mathcal{D}_{m}=\left(\begin{array}{cc}
0 & 1 \\
\lambda^{2}+\frac{m^{2}}{r^{2}} & -\frac{1}{r}
\end{array}\right), \quad \boldsymbol{w}_{m}=\left(\begin{array}{c}
w_{m}^{e, o} \\
\partial_{r} w_{m}^{e, o}
\end{array}\right)
$$

The parity index $e, o$ is dropped hereafter, since the even and odd variables satisfy the same equations. Introduce the transformation operator $\boldsymbol{B}_{m}$, its inverse $\mathcal{B}_{m}^{-1}$ and dispersion free fields $\boldsymbol{v}_{m}=\left(v_{m}^{+}, v_{m}^{-}\right)^{T}$, such that

$$
\boldsymbol{v}_{m}=\boldsymbol{\mathcal { B }}_{m} \boldsymbol{w}_{m}, \quad \boldsymbol{w}_{m}=\mathcal{B}_{m}^{-1} \boldsymbol{v}_{m}, \quad \partial_{r} \boldsymbol{v}_{m}=\boldsymbol{\Lambda} \boldsymbol{v}_{m}
$$

where $\Lambda=c^{-1} \operatorname{diag}\left\{-\partial_{t}, \partial_{t}\right\}$. Since $v_{m}^{+}$and $v_{m}^{-}$satisfy first order one-way wave equations they may be expressed as

$$
v_{m}^{ \pm}(r, t)=v_{m}^{ \pm}\left(r^{\prime}, t \mp\left(r-r^{\prime}\right) / c\right),
$$


where $r^{\prime}$ is some reference radius. Inserting $w_{m}=\mathcal{B}_{m, 11}^{-1} v_{m}^{+}+\mathcal{B}_{m, 12}^{-1} v_{m}^{-}$in (32) and using Laplace transform techniques, the solutions of the ordinary differential equations are obtained as

$$
\begin{aligned}
& \widetilde{\mathcal{B}}_{m, 11}^{-1}=a_{1}(s) \mathrm{I}_{m}(\tilde{\lambda} r) \mathrm{e}^{\left(s c_{i}^{-1}\left(r-r^{\prime}\right)\right)}+b_{1}(s) \mathrm{K}_{m}(\tilde{\lambda} r) \mathrm{e}^{\left(s c_{i}^{-1}\left(r-r^{\prime}\right)\right)}, \\
& \widetilde{\mathcal{B}}_{m, 12}^{-1}=a_{2}(s) \mathrm{I}_{m}(\tilde{\lambda} r) \mathrm{e}^{-\left(s c_{i}^{-1}\left(r-r^{\prime}\right)\right)}+b_{2}(s) \mathrm{K}_{m}(\tilde{\lambda} r) \mathrm{e}^{-\left(s c_{i}^{-1}\left(r-r^{\prime}\right)\right)},
\end{aligned}
$$

where $I_{m}$ and $K_{m}$ are the modified Bessel functions of the first and second kind respectively. Reasonable physical properties of $w$ are that the incoming fields, due to $v_{m}^{-}$, are regular at the origin, while the outgoing fields, due to $v_{m}^{+}$, are regular at infinity. This is accomplished by setting $a_{1}(s)=b_{2}(s)=0$. If we choose $a_{2}(s)=b_{1}(s)=1$ and set $r^{\prime}=0$ for all the fields, the Laplace transforms of the operators $\mathcal{B}_{m}^{-1}$ and $\mathcal{B}_{m}$ are

$\widetilde{\mathcal{B}}_{m}^{-1}=\left(\begin{array}{cc}\mathrm{K}_{m}(\tilde{\lambda} r) \mathrm{e}^{\frac{s r}{c}} & \mathrm{I}_{m}(\tilde{\lambda} r) \mathrm{e}^{-\frac{s r}{c}} \\ \tilde{\lambda} \mathrm{K}_{m}^{\prime}(\tilde{\lambda} r) \mathrm{e}^{\frac{s r}{c}} & \tilde{\lambda} \mathrm{I}_{m}^{\prime}(\tilde{\lambda} r) \mathrm{e}^{-\frac{s r}{c}}\end{array}\right), \widetilde{\mathcal{B}}_{m}=r\left(\begin{array}{cc}\tilde{\lambda} \mathrm{I}_{m}^{\prime}(\tilde{\lambda} r) \mathrm{e}^{-\frac{s r}{c}} & -\mathrm{I}_{m}(\tilde{\lambda} r) \mathrm{e}^{-\frac{s r}{c}} \\ -\tilde{\lambda} \mathrm{K}_{m}^{\prime}(\tilde{\lambda} r) \mathrm{e}^{\frac{s r}{c}} & \mathrm{~K}_{m}(\tilde{\lambda} r) \mathrm{e}^{\frac{s r}{c}}\end{array}\right)$.

In the time domain $\mathcal{B}_{m}^{-1}=\boldsymbol{B}_{m}^{-1} *$ and $\mathcal{B}_{m}=\boldsymbol{B}_{m} *$. The kernel matrices may be written as $[19,20]$

$$
\boldsymbol{B}_{m}^{-1}=\left(\begin{array}{cc}
\Phi_{m}^{+} & \Phi_{m}^{-} \\
L^{-} \Phi_{m}^{+} & L^{+} \Phi_{m}^{-}
\end{array}\right), \quad \boldsymbol{B}_{m}=r\left(\begin{array}{cc}
L^{+} \Phi_{m}^{-} & -\Phi_{m}^{-} \\
-L^{-} \Phi_{m}^{+} & \Phi_{m}^{+}
\end{array}\right)
$$

where $L^{ \pm}=\partial_{r} \pm c^{-1} \partial_{t}$. Using $t^{\prime}=c t / r, \Phi_{m}^{ \pm}$are given by $[19,20]$

$$
\Phi_{m}^{+}=\left\{\begin{array}{ll}
\frac{c}{r} \frac{\mathrm{T}_{m}\left(t^{\prime}+1\right)}{\sqrt{t^{\prime}\left(2+t^{\prime}\right)}} & 0<t^{\prime} \\
0 & t^{\prime}<0
\end{array}, \quad \Phi_{m}^{-}= \begin{cases}(-1)^{m} \frac{c}{r \pi} \frac{\mathrm{T}_{m}\left(t^{\prime}-1\right)}{\sqrt{t^{\prime}\left(2-t^{\prime}\right)}} & t^{\prime} \in(0,2) \\
0 & t^{\prime} \notin(0,2)\end{cases}\right.
$$

where $\mathrm{T}_{m}$ are the Chebyshev polynomials of the first kind. Note that the kernels $\Phi_{m}^{ \pm}$are singular at $t^{\prime}=0$ and that the $\Phi_{m}^{-}$are singular at $t^{\prime}=2$ and have compact support.

There are reasons to comment on the fields $v_{m}^{ \pm}$. Suppose a line source is located at a radius $r=r_{1}$, say. The transformations from the physical fields are such that this source will solely cause fields $v_{m}^{-}$in $r<r_{1}$ and $v_{m}^{+}$in $r>r_{1}$. Hence, reflections from the origin are part of $v_{m}^{-}$for $r<r_{1}$. Hence, the fields $v_{m}^{ \pm}$ are not in- and outgoing fields in the usual sense. Moreover, $v_{0}^{+}(0, t)$ may be interpreted as the magnitude of a point force at the origin, as $\mathrm{K}_{0}\left(\frac{s r}{c}\right)$ is seen to be the Green's function for the modified Helmholtz equation. The present normalization of the transformation is such that $v_{m}^{-}(0, t)=w_{m}(0, t)$, in the case of no sources at the origin. 
There are of course other normalization alternatives that may be a better choice when considering numerical implementation. For the calculations in Section 5.4 we make the choice $a_{2}(s)=b_{1}(s)=\sqrt{s}$.

\section{The Mindlin Plate}

As for the Timoshenko beam, the derivation of the dispersion free transformation will take place in steps. The first step is to decouple the equations obtaining three second order partial differential equations, which is in line with Section 2.2. Up to this point, we do not specify any particular coordinate system in the plane of the plate. However, for the wave splitting operation, the cylindrical geometry is chosen in order to obtain dispersion free radial waves. Hence, the dependent variables are expanded in the angular direction. From here, the derivation of the dispersion free fields is performed in a similar manner as for the membrane, Section 3.

The Mindlin plate equation for a plate of thickness $h$ and with density $\rho$ may be written [17]

$$
\begin{aligned}
& \frac{D}{2}\left\{(1-\nu) \nabla^{2} \boldsymbol{\psi}+(1+\nu) \nabla \nabla \cdot \boldsymbol{\psi}\right\}-\kappa^{2} G h \boldsymbol{\gamma}=\frac{\rho h^{3}}{12} \partial_{t}^{2} \boldsymbol{\psi}, \\
& \kappa^{2} G h \nabla \cdot \boldsymbol{\gamma}=\rho h \partial_{t}^{2} w, \\
& \boldsymbol{\gamma}=\nabla w+\boldsymbol{\psi},
\end{aligned}
$$

where $D=E h^{3} /\left(12\left(1-\nu^{2}\right)\right), E$ is Young's modulus, $G$ is the shear modulus, $\nu$ is Poisson's ratio and $\kappa^{2}$ is the shear coefficient. $w$ is the displacement transverse to the central plane of the plate, that is, in the $z$-direction. $\psi$ is the two-dimensional vector of rotations and $\gamma$ is the two-dimensional vector of shearing angles.

\subsection{Reduction to second order equations.}

In analogy with [17] and [18], we make the ansatz

$$
\boldsymbol{\psi}=\mathcal{A} \nabla w-\mathbf{e}_{z} \times \nabla u_{3}
$$

where $\mathcal{A}$ is an operator which is independent of the spatial coordinates. To separate $w$ and $u_{3}$, we first take the curl of (38), using (38)-(41), which reduces to

$$
\left(\nabla^{2}-\lambda_{3}^{2}\right) u_{3}=0, \quad \lambda_{3}^{2}=c_{3}^{-2} \partial_{t}^{2}+\kappa^{2} / r_{0}^{2}
$$


where $r_{0}=h / \sqrt{12}$ is the radius of gyration of the plate cross section and $c_{3}=\sqrt{G / \rho}$ is the wave front speed.

The equation for $w$ is obtained by taking the divergence of (38), using (38)(41), which turns into the often used equation for the plate

$$
\left(\nabla^{2} \nabla^{2}-\left(c_{1}^{-2}+c_{2}^{-2}\right) \partial_{t}^{2} \nabla^{2}+c_{2}^{-2} r_{0}^{-2} \partial_{t}^{2}+c_{1}^{-2} c_{2}^{-2} \partial_{t}^{4}\right) w=0
$$

Hence, (43) is the two-dimensional counterpart of the beam equation (16), where $c_{1}=\kappa c_{3}$ and $c_{2}=\sqrt{E /\left(\rho\left(1-\nu^{2}\right)\right)}$ are the velocities of the shearing and bending modes respectively. In analogy with (17), (43) can be factorised as

$$
\left(\nabla^{2}-\lambda_{1}^{2}\right)\left(\nabla^{2}-\lambda_{2}^{2}\right) w=0
$$

where the operators $\lambda_{i}^{2}$ are obtained from (18), but with $c_{1}, c_{2}$ and $r_{0}$ for the plate. Introduce new fields $u_{1}$ and $u_{2}$ which, in accordance with (19), gives the set of equations

$$
\left(\nabla^{2}-\lambda_{i}^{2}\right) u_{i}=0, \quad i=1,2,3 .
$$

By using (40)-(42) and (45) the new fields are related to the physical fields through relations similar to $(22)$

$$
\begin{aligned}
w & =u_{1}+u_{2} \\
\boldsymbol{\psi} & =\mathcal{A}_{1} \nabla u_{1}+\mathcal{A}_{2} \nabla u_{2}-\mathbf{e}_{z} \times \nabla u_{3}, \\
\boldsymbol{\gamma} & =\left(1+\mathcal{A}_{1}\right) \nabla u_{1}+\left(1+\mathcal{A}_{2}\right) \nabla u_{2}-\mathbf{e}_{z} \times \nabla u_{3}, \\
\nabla \cdot \boldsymbol{\psi} & =\mathcal{A}_{1} \lambda_{1}^{2} u_{1}+\mathcal{A}_{1} \lambda_{2}^{2} u_{2}, \\
\nabla \times \boldsymbol{\psi} & =-\lambda_{3}^{2} u_{3} \mathbf{e}_{z} .
\end{aligned}
$$

\subsection{Reduction of dependent variables.}

The aim is to reduce the equation system of nine dependent variables to a onedimensional problem. Folkow [21] expressed the state of a plate in a Cartesian coordinate system where the number of dependent variables was reduced to six after performing a Fourier transform in one spatial direction. In the present case, the fields are expanded in the angular direction. For $\eta^{e}=w, u_{1}, u_{2}, \psi_{r}, \gamma_{r}$ and $\eta^{o}=\psi_{\theta}, \gamma_{\theta}, u_{3}$,

$$
\begin{aligned}
& \eta(r, \theta, t)=\eta_{0}^{e}(r, t)+\sum_{m=1}^{\infty}\left(\eta_{m}^{e}(r, t) \cos (m \theta)+\eta_{m}^{o}(r, t) \sin (m \theta)\right) \\
& \eta(r, \theta, t)=\eta_{0}^{o}(r, t)+\sum_{m=1}^{\infty}\left(\eta_{m}^{o}(r, t) \cos (m \theta)-\eta_{m}^{e}(r, t) \sin (m \theta)\right)
\end{aligned}
$$


The even and odd components decouple, so the parity index $e, o$ will be suppressed in the following. Expansions (47)-(48) enable us to eliminate, from the nine first order variables, the ones which are differentiated with respect to the angle $\theta$. Thus, all in all we have six dependent variables for each $m$ and parity.

Define the vectors of the physical variables $\boldsymbol{w}_{m}=\left(w_{m}, \psi_{r, m}, \gamma_{r, m}, \partial_{r} \psi_{r, m}, \psi_{\theta, m}, \partial_{r} \psi_{\theta, m}\right)^{T}$ and the new variables $\boldsymbol{u}_{m}=\left(u_{1, m}, \partial_{r} u_{1, m}, u_{2, m}, \partial_{r} u_{2, m}, u_{3, m}, \partial_{r} u_{3, m}\right)^{T}$. By (46), these fields are related through

$$
\boldsymbol{u}_{m}=\boldsymbol{S}_{m} \boldsymbol{w}_{m}, \quad \boldsymbol{w}_{m}=\mathcal{S}_{m}^{-1} \boldsymbol{u}_{m}
$$

where

$$
\begin{gathered}
\mathcal{S}_{m}^{-1}=\left(\begin{array}{cccccc}
1 & 0 & 1 & 0 & 0 & 0 \\
0 & \mathcal{A}_{1} & 0 & \mathcal{A}_{2} & -\frac{m}{r} & 0 \\
0 & 1+\mathcal{A}_{1} & 0 & 1+\mathcal{A}_{2} & -\frac{m}{r} & 0 \\
\frac{m^{2}}{r^{2}} \mathcal{A}_{1}+\mathcal{V} & -\frac{1}{r} \mathcal{A}_{1} & \frac{m^{2}}{r^{2}} \mathcal{A}_{2}+\frac{2 \tau}{c_{2} r_{0}} \partial_{t}^{2}-\mathcal{V} & -\frac{1}{r} \mathcal{A}_{2} & \frac{m}{r^{2}} & \frac{m}{r} \\
\frac{m}{r} \mathcal{A}_{1} & 0 & \frac{m}{r} \mathcal{A}_{2} & 0 & 0 & 1 \\
-\frac{m}{r^{2}} \mathcal{A}_{1} & \frac{m}{r} \mathcal{A}_{1} & -\frac{m}{r^{2}} \mathcal{A}_{2} & \frac{m}{r} \mathcal{A}_{2} & -\left(\lambda_{3}^{2}+\frac{m^{2}}{r^{2}}\right) & -\frac{1}{r}
\end{array}\right), \\
\mathcal{S}_{m} \\
\left.\begin{array}{cccccc}
1-\mathcal{N}_{1} & -\frac{1}{r} \mathcal{N}_{4} & 0 & -\mathcal{N}_{4} & \frac{m}{r} \mathcal{N}_{4} & 0 \\
0 & -1-\mathcal{N}_{2}+\frac{m^{2}}{r^{2}} \mathcal{M}_{4} & 1-\mathcal{N}_{3} & 0 & -\frac{m}{r^{2}} \mathcal{M}_{4} & -\frac{m}{r} \mathcal{M}_{4} \\
\mathcal{N}_{1} & \frac{1}{r} \mathcal{N}_{4} & 0 & \mathcal{N}_{4} & -\frac{m}{r} \mathcal{N}_{4} & 0 \\
0 & \mathcal{N}_{2}-\frac{m^{2}}{r^{2}} \mathcal{M}_{4} & \mathcal{N}_{3} & 0 & \frac{m}{r^{2}} \mathcal{M}_{4} & \frac{m}{r} \mathcal{M}_{4} \\
0 & \frac{m}{r} \mathcal{M}_{3} & 0 & 0 & -\frac{1}{r} \mathcal{M}_{3} & -\mathcal{M}_{3} \\
\frac{m}{r} \mathcal{M}_{1} & -\frac{m}{r^{2}} \mathcal{M}_{2} & 0 & -\frac{m}{r} \mathcal{M}_{2} & 1+\frac{m^{2}}{r^{2}} \mathcal{M}_{2} & 0
\end{array}\right) .
\end{gathered}
$$

The operators $\mathcal{N}_{i}$ and $\mathcal{A}_{i}$ are obtained from the beam case in Section 2.2 but with $c_{1}, c_{2}$ and $r_{0}$ for the plate. The $\mathcal{M}_{i}$ are given in Appendix A. Note that $u_{3,0}$ decouples from $u_{1,0}$ and $u_{2,0}$, as found also in [21]. The same kind of decoupling also occurs when $r \rightarrow \infty$, in which case $u_{1, m}$ and $u_{2, m}$ transform as in the Timoshenko case.

So far we have derived a transformation that takes the Fourier components of the physical fields, $\boldsymbol{w}_{m}$ and gives a new set of variables $\boldsymbol{u}_{m}$, which obey three decoupled second order wave equations, with different wave front speeds. We now perform the wave splitting on each of these wave equations, giving six one-way wave equations, with the additional condition that the one-way wave equations shall be free of dispersion. 


\subsection{Transformation to dispersion free fields.}

The transformation to dispersion free fields is performed in the same manner as for the membrane in Section 3. For each Fourier component, equation (45) may be written

$$
\partial_{r} \boldsymbol{u}_{i, m}=\mathcal{D}_{i, m} \boldsymbol{u}_{i, m}, \quad \mathcal{D}_{i, m}=\left(\begin{array}{cc}
0 & 1 \\
\lambda_{i}^{2}+\frac{m^{2}}{r^{2}} & -\frac{1}{r}
\end{array}\right), \quad \boldsymbol{u}_{i, m}=\left(\begin{array}{c}
u_{i, m} \\
\partial_{r} u_{i, m}
\end{array}\right)
$$

Introduce the transformation operator $\mathcal{B}_{i, m}$, its inverse $\mathcal{B}_{i, m}^{-1}$ and dispersion free fields $\boldsymbol{v}_{i, m}=\left(v_{i, m}^{+}, v_{i, m}^{-}\right)^{T}$ such that

$$
\boldsymbol{v}_{i, m}=\mathcal{B}_{i, m} \boldsymbol{u}_{i, m}, \quad \boldsymbol{u}_{i, m}=\mathcal{B}_{i, m}^{-1} \boldsymbol{v}_{i, m}, \quad \partial_{r} \boldsymbol{v}_{i, m}=\boldsymbol{\Lambda}_{i} \boldsymbol{v}_{i, m}
$$

where $\boldsymbol{\Lambda}_{i}=c_{i}^{-1} \operatorname{diag}\left\{-\partial_{t}, \partial_{t}\right\}$. The following steps are identical to the membrane case and equations (33)-(36) holds for the the fields $u_{i}, i=1,2,3$, by adding the index $i$ to $c, \lambda, a_{1}, a_{2}, b_{1}, b_{2}, \mathcal{B}_{m}, \mathcal{B}_{m}^{-1}, \Phi_{m}^{ \pm}$. The coefficients $a_{i, 1} \ldots b_{i, 2}$ are chosen on the same basis as for the membrane noting that the leading terms of the $\lambda_{i}$ are of the same order as in the membrane case. Hence, $a_{i, 1}=b_{i, 2}=0$ and $a_{i, 2}=b_{i, 1}=1$.

The inverse Laplace transforms of these elements are not as easy as in the case of the membrane. There is, however, at least one case where an explicit expression for the elements of the matrices exist, namely when $i=3$ and $m=0[20]$. Then,

$$
\Phi_{3,0}^{+}=\left\{\begin{array}{ll}
\frac{c_{3} \cos \left(\alpha \sqrt{t^{\prime}\left(2+t^{\prime}\right)}\right)}{r \sqrt{t^{\prime}\left(2+t^{\prime}\right)}} & 0<t^{\prime} \\
0 & t^{\prime}<0
\end{array}, \Phi_{3,0}^{-}=\left\{\begin{array}{ll}
\frac{c_{3} \cosh \left(\alpha \sqrt{t^{\prime}\left(2-t^{\prime}\right)}\right)}{r \pi \sqrt{t^{\prime}\left(2-t^{\prime}\right)}} & t^{\prime} \in(0,2) \\
0 & t^{\prime} \notin(0,2)
\end{array},\right.\right.
$$

where $\alpha=\kappa r / r_{0}$ and $t^{\prime}=c_{3} t / r$. In conformity with the membrane case, the kernels $\Phi_{3,0}^{ \pm}$are singular at $t^{\prime}=0$ and $\Phi_{3,0}^{-}$is singular at $t^{\prime}=2$ and has compact support. Note that $\Phi_{3,0}^{-}$grows exponentially with increasing radius, in contradistinction to the case of the $u_{3}$-mode in Cartesian coordinates [21]. For the other cases, the behaviour in the time domain for small times can be obtained approximately by studying the asymptotics of the elements of $\tilde{\mathcal{B}}_{i, m}$ and $\widetilde{\mathcal{B}}_{i, m}^{-1}$ for large values of the Laplace variable, see Appendix B. From the asymptotic expansions it is indicated that all the kernels $\Phi_{i, m}^{-}$have compact support.

With $\boldsymbol{v}_{m}=\left(v_{1, m}^{+}, v_{1, m}^{-}, v_{2, m}^{+}, v_{2, m}^{-}, v_{3, m}^{+}, v_{3, m}^{-}\right)^{T}$ and $\mathcal{B}=\operatorname{diag}\left(\boldsymbol{\mathcal { B }}_{1, m}, \mathcal{B}_{2, m}, \mathcal{B}_{3, m}\right)$, the combined transform is

$$
\boldsymbol{v}_{m}=\mathcal{B}_{m} \mathcal{S}_{m} \boldsymbol{w}_{m}, \quad \boldsymbol{w}_{m}=\mathcal{S}_{m}^{-1} \mathcal{B}_{m}^{-1} \boldsymbol{v}_{m}
$$




\section{Application to an inhomogeneous membrane}

\subsection{The dynamic equations}

We now consider the case when the mass per unit area of the membrane varies as a function of the radius. Hence, the wave speed will be a function of $r$. We use the same transformation matrices $\mathcal{B}_{m}$ and $\mathcal{B}_{m}^{-1}$ as in Section 3, however with a varying velocity $c(r)$. Insertion of $\boldsymbol{w}_{m}=\boldsymbol{B}_{m}^{-1} \boldsymbol{v}_{m}$, into (32), gives

$$
\partial_{r} \boldsymbol{v}_{m}=\left(\mathcal{B D}_{m} \mathcal{B}_{m}^{-1}-\mathcal{B} \partial_{r} \mathcal{B}_{m}^{-1}\right) \boldsymbol{v}_{m}
$$

In order to use the results from the homogeneous membrane in Section 3, we introduce the following decomposition

$$
\partial_{r} \mathcal{B}_{m}^{-1}=\left.\partial_{r}\right|_{c(r)=\text { const. }} \mathcal{B}_{m}^{-1}+c^{\prime}(r) \partial_{c(r)} \mathcal{B}_{m}^{-1}
$$

Hence, the dynamics of the split fields becomes

$$
\partial_{r} \boldsymbol{v}_{m}=\left(\boldsymbol{\Lambda}+\mathcal{L}_{m}\right) \boldsymbol{v}_{m}, \quad \boldsymbol{\Lambda}=c(r)^{-1} \partial_{t} \operatorname{diag}(-1,1), \quad \mathcal{L}_{m}=-c^{\prime}(r) \boldsymbol{B}_{m} \partial_{c(r)} \mathcal{B}_{m}^{-1}
$$

Thus, at each point we perform the wave splitting with respect to the local wave speed. In the Laplace domain, the operator elements of $\mathcal{L}_{m}$ are

$$
\begin{aligned}
\widetilde{\mathcal{L}}_{m, 11} & =\frac{c^{\prime}}{c} \zeta^{2}\left(\mathrm{I}_{m}(\zeta)-\mathrm{I}_{m}^{\prime}(\zeta)\right)\left(\mathrm{K}_{m}(\zeta)+\mathrm{K}_{m}^{\prime}(\zeta)\right)-m^{2} \mathrm{I}_{m}(\zeta) \mathrm{K}_{m}(\zeta), \\
\widetilde{\mathcal{L}}_{m, 12} & =\frac{c^{\prime}}{c}(\zeta)^{2}\left(\mathrm{I}_{m-1}(\zeta) \mathrm{I}_{m+1}(\zeta)-\mathrm{I}_{m}^{2}(\zeta)\right) \mathrm{e}^{-2 \zeta} \\
\widetilde{\mathcal{L}}_{m, 21} & =\frac{c^{\prime}}{c}(\zeta)^{2}\left(-\mathrm{K}_{m-1}(\zeta) \mathrm{K}_{m+1}(\zeta)+\mathrm{K}_{m}^{2}(\zeta)\right) \mathrm{e}^{2 \zeta} \\
\widetilde{\mathcal{L}}_{m, 22} & =-\widetilde{\mathcal{L}}_{m, 11}
\end{aligned}
$$

where $c=c(r), c^{\prime}=c^{\prime}(r)$ and $\zeta=s r / c$. These elements can be obtained in the time domain by taking the inverse Laplace transformation of each modified Bessel function separately, applying the convolution theorem and finally differentiate twice with respect to time. They then have the form

$$
\mathcal{L}_{m, k l} v^{ \pm}=L_{m, k l} * v_{m}^{ \pm}=\int_{0^{-}}^{t^{+}} L_{m, k l}\left(r, t^{\prime}\right) v_{m}^{ \pm}\left(r, t-t^{\prime}\right) \mathrm{d} t^{\prime}, \quad k, l=1,2 .
$$

So the dynamics for the split fields, (55), become

$$
\begin{aligned}
& \partial_{r} v_{m}^{+}=-c^{-1} \partial_{t} v_{m}^{+}+L_{m, 11} * v_{m}^{+}+L_{m, 12} * v_{m}^{-}, \\
& \partial_{r} v_{m}^{-}=c^{-1} \partial_{t} v_{m}^{-}+L_{m, 21} * v_{m}^{+}-L_{m, 11} * v_{m}^{-}
\end{aligned}
$$

Thus, for an inhomogeneous region the split fields satisfy coupled partial integro-differential equations. From here, one can exploit various time domain 
methods, such as the imbedding method, Green's functions and the propagator technique, to solve direct and inverse problems. In the example considered here we utilise the imbedding method. In Section 5.3 explicit expressions for the $L_{m, i j}$-kernels are given in the case $m=0$.

\subsection{The imbedding equation}

We seek an equation for the operator that, for a certain $r$ in the inhomogeneous region, relates the internal fields to each other. Due to linearity, causality and time translation invariance this relation has the form

$$
v_{m}^{+}(r, t)=\int_{0^{-}}^{t^{+}} R_{m}\left(r, t^{\prime}\right) v_{m}^{-}\left(r, t-t^{\prime}\right) \mathrm{d} t^{\prime}
$$

The kernel $R_{m}(r, t)$ will be referred to as the reflection kernel as usual, although $v_{m}^{ \pm}$are not physical wave fields traveling inwards and outwards in the usual sense. To obtain an equation for $R_{m}(r, t), v_{m}^{+}$is eliminated from (61) by insertion of (62). After use of both equations, partial integration and the causality of $R_{m}(r, t)$ and $v_{m}^{-}$, the equation for $R$ is obtained as

$$
\left(\partial_{r}+\frac{2}{c} \partial_{t}\right) R_{m}-2 L_{m, 11} * R_{m}+R_{m} * L_{m, 21} * R_{m}-L_{m, 12}=0 .
$$

In the following the analysis will be restricted to the case $m=0$, so the index

$m$ is suppressed hereafter. It is also assumed that $c(r)$ is continuous, while $c^{\prime}(r)$ is piecewise continuous.

\subsection{Certain known components of the reflection kernel for the case $m=0$}

In the time domain the kernels elements of $\mathcal{L}_{0}$ become, after lengthy calculations,

$$
\begin{aligned}
& L_{11}=\frac{c^{\prime}}{c} \begin{cases}b_{11}\left\{\frac{\mathrm{E}\left(d_{11}^{2}\right)}{d_{11}^{2}\left(1-d_{11}^{2}\right)}-\frac{\mathrm{K}\left(d_{11}^{2}\right)}{d_{11}^{2}},\right\} & t<\frac{2 r}{c}, \\
\frac{b_{11}}{d_{11}\left(d_{11}^{-2}\right)} \frac{t-d_{11}^{2}}{1}, & t>\frac{2 r}{c},\end{cases} \\
& L_{12}=\frac{c^{\prime}}{c} \begin{cases}-\frac{\delta(t)}{2 \pi}+\mathrm{H}(t) b_{12}\left\{(4 r-c t) \mathrm{E}\left(d_{12}^{-1}\right)+2(2 r-c t) \mathrm{K}\left(d_{12}^{-1}\right)\right\}, & t<\frac{2 r}{c}, \\
-\frac{\delta\left(\frac{4 r}{c}-t\right)}{2 \pi}+\mathrm{H}\left(\frac{4 r}{c}-t\right) b_{12}\left\{c t \mathrm{E}\left(d_{12}\right)+2(2 r-c t) \mathrm{K}\left(d_{12}\right)\right\}, & t>\frac{2 r}{c},\end{cases} \\
& L_{21}=\frac{c^{\prime}}{c}\left\{-\frac{\pi}{2} \delta(t)-b_{21}\left\{(4 r+c t) \mathrm{E}\left(d_{21}^{-1}\right)-2(2 r+c t) \mathrm{K}\left(d_{21}^{-1}\right)\right\}\right\},
\end{aligned}
$$


where $\mathrm{H}$ is the Heavyside step function and $\mathrm{K}, \mathrm{E}$ are the complete elliptic integrals of the first and second kind respectively. The $b_{k l}$ and $d_{k l}$ are given by

$$
\begin{aligned}
& b_{11}=\frac{c}{2 \pi r}, \quad b_{12}=\frac{4 r^{2}}{\pi^{2} t(c t-4 r)(c t-2 r)^{2}}, \quad b_{21}=\frac{4 r^{2}}{t(c t+4 r)(c t+2 r)^{2}}, \\
& d_{11}=\frac{c t}{2 r}, \quad d_{12}=\left(1-\frac{4 r}{c t}\right)^{2}, \quad d_{21}=\left(1+\frac{4 r}{c t}\right)^{2} .
\end{aligned}
$$

The kernels of the coupling operator are defined in terms of E and K, with algebraic coefficients and arguments, which are singular. In addition, K has a logarithmic singularity when the argument approaches unity. The kernels also contain step functions and Dirac's delta functions. It implies that $R(r, t)$ may contain these types of singularities as well.

Equation (63) thus contains terms of these kinds. Billger and Folkow [2] give a proof, which, in effect, states that multiplicative and convolutional parts of (62) must satisfy (62) independently. The coefficients for collected Dirac's delta functions in (63) having the same characteristic then must be zero. It may also be shown that coefficients for power and logarithmic singularities must be zero. This allows for analytical determination of some of the singularities and distributions of the reflection kernel.

For continuous $c(r), R(r, t)$ contains no Dirac's delta functions. Hence, the reflection kernel is decomposed according to

$$
\begin{aligned}
R(r, t)= & R_{1}(r, t)+\sum[R]_{i}(r) \mathrm{H}\left(t-\beta_{i}(r)\right)+\sum g_{i}(r)\left(t-\gamma_{i}(r)\right)^{-1} \\
& +\sum f_{i}(r) \ln \left(\left|t-\gamma_{i}(r)\right|\right)+\sum h_{i}(r)\left(t-\gamma_{i}(r)\right) \ln \left(\left|t-\gamma_{i}(r)\right|\right),
\end{aligned}
$$

where $R_{1}(r, t)$ is the bounded and smooth part of $R(r, t)$. The jump discontinuities give rise to Dirac's delta functions when inserted into (63), but so does convolutions of power singularities, consider for instance [22]

$$
\int_{0}^{t} \frac{\mathrm{d} t^{\prime}}{\left(t^{\prime}-t_{1}\right)\left(t-t^{\prime}-t_{0}\right)}=\frac{1}{t-t_{0}-t_{1}} \ln \left|\frac{\left(t-t_{0}\right)\left(t-t_{1}\right)}{t_{0} t_{1}}\right|-\pi^{2} \delta\left(t-t_{1}-t_{0}\right),
$$

where $t_{0}, t_{1}>0$. There is however one jump discontinuity that can be determined at this point, since these latter contributions propagate along "later" characteristics. The kernel $L_{12}$ contains $-\frac{c^{\prime}}{2 \pi c} \delta(t)$. Cancellation is only possible if $R$ has a jump discontinuity $[R]_{0}$ according to (65), given by

$$
[R]_{0}=-c^{\prime} / 4 \pi, \quad \beta_{0}(r)=0
$$

Now, consider the power singularities. By studying $L_{k l}, k, l=1,2$, in the neighbourhood of the singularities, i.e. by examining expansions about singular points, the coefficients of the singular parts can be identified. After insertion of $R$ into (63), singular terms of the same order propagating along the 
same characteristics are collected. The coefficients for these must vanish independently, which makes it possible to determine the necessary singularities of $R$.

For power singularities of order one the convolution integral (62) is interpret as a CPV integral since it is valid for all $t$. The same holds for convolutions in (63). A power singularity of order two is found in $L_{12}$. The only term that can cancel this term comes from the principal part of (63). Thus,

$$
-g_{0}\left(t-\gamma_{0}\right)^{-2}\left(2 / c-\gamma_{0}^{\prime}\right)-\left(-2 c^{\prime} r \pi^{-2}(c t-2 r)^{-2}\right)=0,
$$

which gives $\gamma_{0}(r)=2 r / c(r)$ and $g_{0}(r)=g_{0}=\pi^{-2}$. Investigation of the other terms in (63) shows that there are no further singularities in $R(r, t)$ along that characteristic, $f_{0}(r)=h_{0}(r)=0$, but from inspection of the principal part it is seen that there may be power singularities propagating along $t=\gamma_{i>0}(r)$, where

$$
\gamma_{i>0}(r)=\int_{0}^{r} \frac{2 \mathrm{~d} r^{\prime}}{c\left(r^{\prime}\right)}+C_{i}^{\gamma}
$$

To determine these, consider a case where $c$ is constant for $r<r^{\prime}$ and a function of $r$ on the outside. Then $R\left(r<r^{\prime}, t\right)=0$. From continuity considerations for $v^{ \pm}$and (62) it follows that there must be a power singularity $g_{1}\left(r^{\prime}\right)=-g_{0}$, that propagates from the point $(r, t)=\left(r^{\prime}, \gamma_{0}\left(r^{\prime}\right)\right)$ along the characteristic $t=\gamma_{1}(r)$, where $\gamma_{1}(r)$ is given by (68) with $C_{1}^{\gamma}=0$, see II in Figure 1. For continuous $c$, there are no further power singularities. From the other terms in (63) we may conclude also that $g_{1}(r)=-g_{0}$ and that $f_{1}(r)$ satisfy $(2 \pi)^{2} \partial_{r} f_{1}=\left(c^{\prime}\right)^{2} / c-c^{\prime} / r$.

Since (63) is non-linear in $R$ and the coupling kernels, as well as $R$, are singular there are Dirac's delta functions propagating also along $t=2 \gamma_{0}, 2 \gamma_{1}, \gamma_{0}+\gamma_{1}$. It turns out that, when each of these terms in (63) are collected, including $-\frac{c^{\prime}}{2 \pi c} \delta\left(t-\frac{4 r}{c}\right)$ from $L_{12}$, the only one with a non-vanishing coefficient propagates along $t=2 \gamma_{1}(r)$ and has the coefficient $c^{\prime} /(2 \pi c)$. This necessitates another jump discontinuity in $R$ along $t=\beta_{1}(r)=2 \gamma_{1}(r)$, which is given by

$$
[R]_{1}\left(2 / c-2 \gamma_{1}^{\prime}\right)+c^{\prime} /(2 \pi c)=0 .
$$

Thus, $[R]_{1}=-[R]_{0}$. There may be additional jump discontinuities $[R]_{i>1}$ propagating along $t=\beta_{i>1}(r)$, where $\beta_{i>1}(r)$ is given by the right hand side of (68). These are initiated where $c^{\prime}$ has jump discontinuities, see I and III in Figure 1 . From considerations based on the continuity of $v^{ \pm}$and equation (63), initial values and differential equations for these additional jump discontinuities may be derived. The additional logarithmic singularities that these jump discontinuities lead to cancel out in (63). Hence, they do not imply further singularities in $R$. There are, however, additional singularities in individual terms in (63) and they run along characteristics such as $t=\gamma_{1}+\beta_{i>0}, \gamma_{2}+\beta_{i>0}$. 
Finally, we note that $\gamma_{1}(r)$ is the true travel time from radius $r$ to the origin and back, while $\gamma_{0}(r)$ is the travel time with the constant wave speed $c(r)$ everywhere, i.e. the travel time for the homogeneous case.

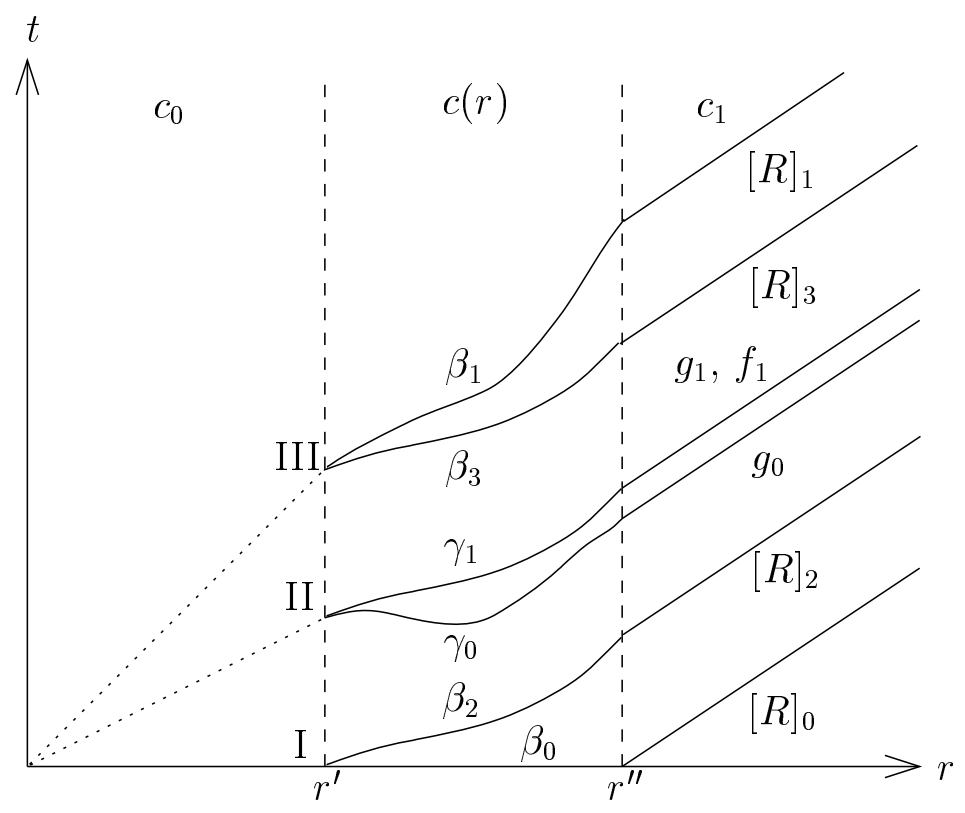

Fig. 1. Characteristics for an inhomogeneous ring with $c^{\prime}(r)$ discontinuous at the boundaries. Note that $[R]_{0}$ runs along $t=0$ for $r^{\prime}<r<r^{\prime \prime}$.

\subsection{Numerical example}

In this section we present numerical results from simulated direct scattering problems for inhomogeneous membranes. All results concern the case with circular symmetry; $w(r, \theta, t)=w_{0}(r, t)$, see Section 3. We will first give an outline of the numerical implementation of the radial wave splitting and the computation of the reflection kernel. The direct problem is then considered for three wave speed profiles, where the result is compared to a FE solution.

\subsubsection{Numerical implementation of the wave splitting and the reflection ker- nel}

As was mentioned at the end of Section 3, one may consider other normalizations of the transforms than the one presented there. Here, we make the choice $a_{2}(s)=b_{1}(s)=\sqrt{s}$. This makes the transform operators more suited for numerical implementation in the time domain, see Appendix A. The kernels are still singular but integrable, interpreted as CPV integrals. This renormalization does not influence the coupling terms (64), and hence not the 
dynamics of the split fields, (61). As a consequence, the reflection operator is not altered.

In Section 5.2 it was demonstrated how parts of the reflection kernel could be determined analytically. The remaining part of $R$ may then be calculated numerically with simple methods, taking into consideration the known singularities. Here, only a subset of the analytical information in Section 5.2 has been utilised, namely the information about the power singularities traveling along $t=\gamma_{0}$ and $t=\gamma_{1}$ and the jump discontinuity $[R]_{0}$. The rest of the jumps and singularities are being part of the numerical solution, and have thus been handled with less accuracy. The reason for this is, that in many scattering situations, the main objective is to be able to determine the fields up to and past one travel time through the inhomogeneous medium and back. This is for example the case when studying inverse problems, where information about the varying media is to be recovered from reflection data. The reflection kernel is therefore divided into a regular part, $R^{\text {reg }}$, and a singular part, whereby we mean that the regular part does not contain power singularities and the jump discontinuity $[R]_{0}$. The regular part is then calculated numerically by means of finite differences.

Since all terms in the imbedding equation contain singularities it is inevitable to have to perform convolutions with singular kernels numerically. This problem has been solved by replacing all singularities with discrete functions that give an approximate result when being integrated, see Appendix C.

Travel time coordinates are introduced to simplify the calculations. If the membrane is homogeneous outside a radius $r=r_{1}$ say, the non-dimensional travel time coordinates are

$$
\xi(r)=\frac{1}{\Xi} \int_{0}^{r} \frac{\mathrm{d} r^{\prime}}{c\left(r^{\prime}\right)}, \quad \eta=t / 2 \Xi, \quad \Xi=\int_{0}^{r_{1}} \frac{\mathrm{d} r^{\prime}}{c\left(r^{\prime}\right)} .
$$

This makes the characteristic equation $t=\gamma_{1}(r)$ become $\eta=\xi$ in nondimensional space. By introducing $L_{12}^{\text {mod }}$ as $L_{12}$ without the power singularity of order two and the first Dirac's delta function at $t=0,(63)$ may be written

$$
\left(\partial_{\xi}+\partial_{\eta}\right) R^{\mathrm{reg}}-2 L_{11} * R+R * L_{21} * R-L_{12}^{\mathrm{mod}}=0 .
$$

Then, (70) does not contain the power singularity of order two and no Dirac's delta functions for $t<2 \min \left(\gamma_{0}, \gamma_{1}\right)$. The inhomogeneous region is then divided into $N$ subintervals and $R^{\text {reg }}(1, s)$ is solved numerically from knowledge of the coupling terms and $R(x, 0)$, which is known from the jump condition (67). The integrals are all approximated with the trapezoidal rule, where the logarithmic singularities and the power singularities of order one are represented according to Appendix C. 


\subsubsection{Comparisons with FE solutions.}

Solutions obtained by means of the commercial software FEMLAB are used for comparisons. The membranes that are considered are of radius $r=r_{2}$, with a possible region of inhomogeneity from the origin to $r=r_{1}<r_{2}$. In all the examples discussed below, the outer radii $r_{2}$ are large enough so that no reflections from these boundaries have to be considered. The membranes are initially at rest, and waves are generated at the boundaries according to

$$
w\left(r_{2}, t\right)= \begin{cases}\sin ^{2}(5 t), & t \in(0, \pi / 5), \\ 0, & t \notin(0, \pi / 5) .\end{cases}
$$

We start by noting that the properties of the wave splitting are such that no $v^{+}$-waves are generated when boundary condition (71) is applied to a homogeneous membrane. The $v^{-}$-waves may then be computed solely from knowledge of $w$. As $v^{-}$propagates without dispersion, it is straightforward to obtain $w(r, t)$ and $\partial_{r} w(r, t)$ everywhere in the membrane using the transformations. When compared to the solutions using the FE solver, the fields $w$ and $w_{\mathrm{FE}}$ agree very well. However, the $w$-solutions were obtained much quicker.

For the inhomogeneous membranes, the objective is to obtain the fields at the outer boundaries of the inhomogeneous regions, $r=r_{1}$. Using the FE solution, $v_{\mathrm{FE}}^{+}\left(r_{1}, t\right)$ and $v_{\mathrm{FE}}^{-}\left(r_{1}, t\right)$ are transformed from $w_{\mathrm{FE}}\left(r_{1}, t\right)$ and its spatial derivative. $v^{+}\left(r_{1}, t\right)$ may also be computed from $v_{\mathrm{FE}}^{-}\left(r_{1}, t\right)$ by means of $(62)$ and is denoted $v_{R}^{+}\left(r_{1}, t\right)$. Comparisons of $v_{R}^{+}$and $v_{\mathrm{FE}}^{+}$are made, where the time is measured from when the incoming waves reach $r=r_{1}$.

Note that it is possible to calculate $v^{-}\left(r_{2}, t\right)$ over a finite time interval from knowledge of only $w\left(r_{2}, t\right)$, using $v^{+}\left(r_{2}, t\right)=0$ as in the homogeneous case. This solution, which is valid up until the reflected fields from the inhomogeneity reach $r=r_{2}$, may then be used at $r=r_{1}$ instead of $v_{\mathrm{FE}}^{-}\left(r_{1}, t\right)$. So, the fields $v^{-}\left(r_{1}, t\right)$ are the same no matter the inhomogeneity, over a finite time interval. This means that $v^{-}$for the homogeneous case can be used in the inhomogeneous cases over this finite time interval.

5.4.2.1 Interior with a lower speed of propagation. Here the wave speed profile has a linear variation, where $c=0.5$ for $r<0.5$ and $c=1$ for $r>0.7=r_{1}$.

We first take a look at the different components of the wave field in Figure 2. By setting $v_{\mathrm{FE}}^{+}=0$ and using the wave splitting transformation we obtain the wave field for a homogeneous membrane, $w_{\mathrm{FE}}^{-}$. Similarly, by setting $v_{\mathrm{FE}}^{-}=0$ we obtain the wave field $w_{\mathrm{FE}}^{+}$, which is the difference between the physical fields for a homogeneous membrane and an inhomogeneous membrane. As is seen in 
Figure 2, $w_{\mathrm{FE}}=w_{\mathrm{FE}}^{+}+w_{\mathrm{FE}}^{-}$, as expected. A rough estimation of the wave field in an inhomogeneous membrane is obtained by approximating the reflection kernel with the known power singularities. The compensation for the reflection at the origin is then taken care of while the reflections at the inhomogeneities, which are small in comparison to the total field, are neglected. In Figure 2 this field is denoted by $w_{\text {SING }}$, and it is seen to agree remarkably well.

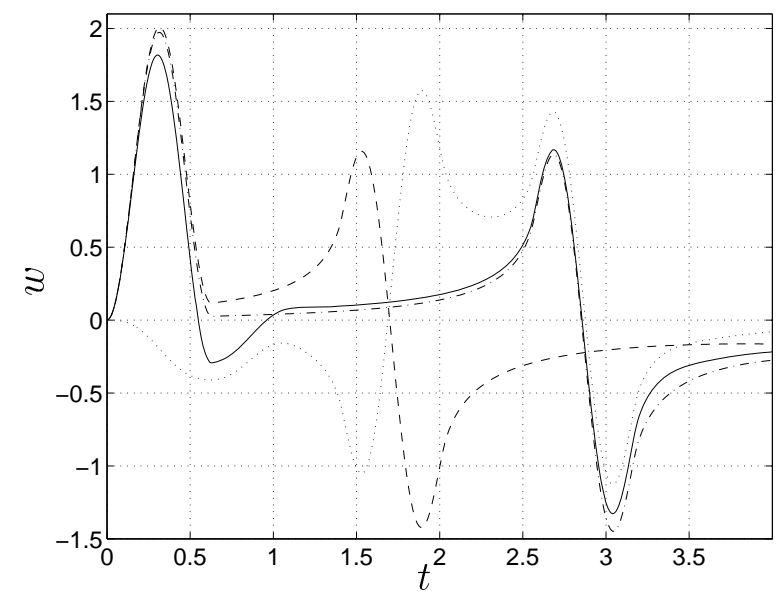

Fig. 2. Different components of $w$ at $r=r_{1}$. (dash-dotted) $w_{\mathrm{SING}}$, (dashed) $w_{\mathrm{FE}}^{-}$, (solid) $w_{\mathrm{FE}}$, (dotted) $w_{\mathrm{FE}}^{+}$.

We now turn to the comparisons. For this wave speed profile we have that $\gamma_{0}(0.7)=1.4$ and $\gamma_{1}(0.7) \approx 2.55$. As is seen in Figure $3(\mathrm{a})$, the error is small up to approximately $t=2.8$, where the solution is first affected by the distributions propagating along $t=2 \gamma_{0}(r)$. The deviation between $t \approx 0.6$ and $t \approx 1.1$ is due to the jump discontinuity, which propagates along $t=\beta_{2}$. The convergence is not that clear in this region since it depends on both the location of the jump discontinuity as well as the approximation of it. It is rather the absolute value of the differences between successive approximations that converges to zero. The structure of the error for other times is due to convolutions with power singularities and the transformation from the physical fields to the split fields. Note that we compare with $1 \%$ of $v_{\mathrm{FE}}^{+}$.

5.4.2.2 Interior with a higher speed of propagation Here, the wave speed profile has a linear variation where $c=2$ for $r<0.5$ and $c=1$ for $r>0.7=r_{1}$. We then have that $\gamma_{0}(0.7)=1.4$ and $\gamma_{1}(0.7) \approx 0.77$. As is seen in Figure $3(\mathrm{~b})$, the error is small up to approximately $t=1.3$. The time of the first influence from distributions propagating along $t=2 \gamma_{i}(r)$ and $t=\gamma_{0}(r)+\gamma_{1}(r)$ is, in this case, $t \approx 1.27$.

5.4.2.3 Stratified interior In this case $r_{1}=1$ and inside there are several layers where the wave speed is linearly varying between the radii $r=$ 


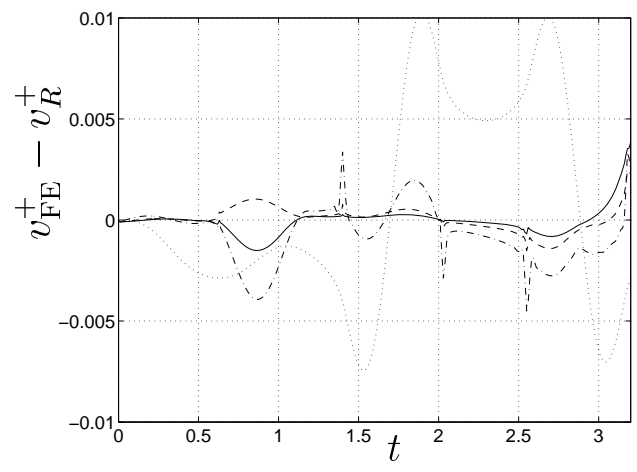

(a) Slow interior.

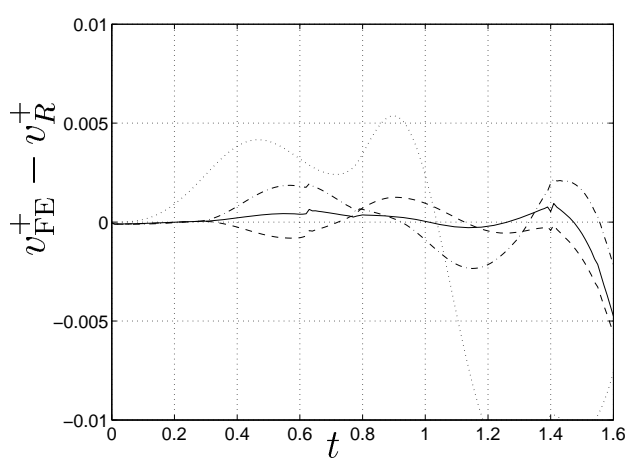

(b) Fast interior.

Fig. 3. The difference $v_{\mathrm{FE}}^{+}-v_{R}^{+}$for slow and fast interior at $r=r_{1}$. (dash-dotted) $N=100$, (dashed) $N=200$, (solid) $N=400$, (dotted) $1 \%$ of $v_{\mathrm{FE}}^{+}$.

$0,0.2,0.5,0.7,1$. The wave speeds at these radii are $c=1.25,1.25,1.2,1.1,1$ respectively. At the boundary of the inhomogeneous region $\gamma_{0}(1)=2$ and $\gamma_{1}(1) \approx 1.73$. From Figure $4(\mathrm{a})$ it is seen that the error is small for times up to approximately $t=2$. The first influence from the "later" characteristics occur for $t \approx 2.05$.

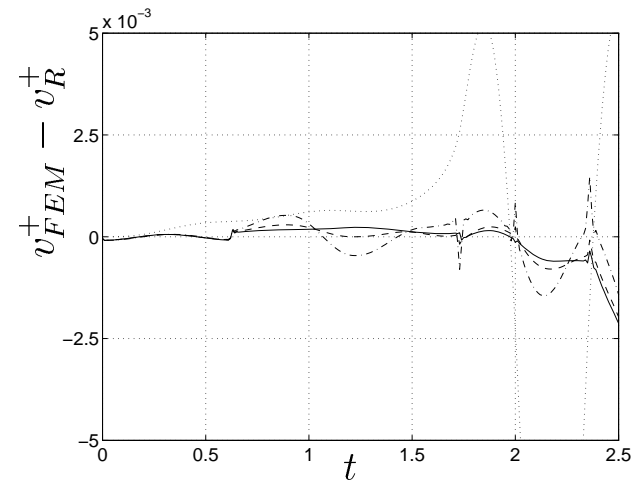

(a) Stratified interior.

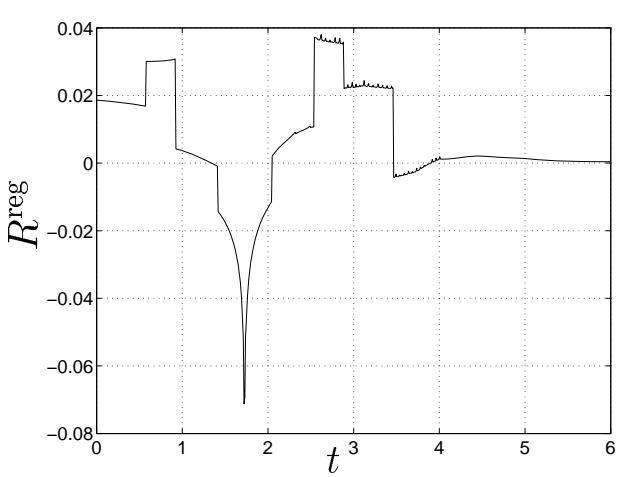

(b) The regular part of $R$.

Fig. 4. The difference $v_{\mathrm{FE}}^{+}-v_{R}^{+}$and the regular part of $R$ for stratified interior at $r=r_{1}$. In (a), (dash-dotted) $N=100$, (dashed) $N=200$, (solid) $N=400$, (dotted) $1 \%$ of $v_{\mathrm{FE}}^{+}$.

The regular part of the reflection kernel, including the logarithmic singularity, propagating along $t=\gamma_{1}$, is shown in Figure 4(b) for the stratified case. Here, some of the numerical difficulties are apparent around time $t=2.5$. 


\section{Conclusions}

This paper deals with wave splittings that transform to one-way wave equations. For the radial membrane and the radial Mindlin plate, which both exhibit directly geometric dispersion, the splitting was obtained using nonstandard methods. This resulted in dispersion free split fields in a straightforward way. This feature could also be exploited for other structures that do not require the presented method to obtain a wave splitting, such as the Timoshenko beam. The main advantage of deriving dispersion free wave splittings is that when the split fields at some point are determined, translation through homogeneous regions is trivial. This property can be utilised when solving inverse problems since one then do not want to waste computational resources on homogeneous regions, especially when the fields are measured far from the inhomogeneous region. But, as is demonstrated in the case of the Timoshenko beam, this is done to the price of a wave splitting transformation that is more resource demanding than the original one.

The method agrees with the extension of a previous wave splitting for the Timoshenko beam. For the radial membrane the transformation is a decomposition into the field from a source at the origin and the regular part of the physical field at the origin. (This decomposition is in analogy with, for instance, the T matrix method. See, e.g., Ref. [23] which incidentally gives the first time domain formulation of the $\mathrm{T}$ matrix method.) By combining these two cases, it is indicated how the wave splitting transformation for the Mindlin plate may be obtained.

To validate the method for inhomogeneous media the inhomogeneous membrane has been investigated by means of the invariant imbedding technique for a direct problem. The results from a numerical implementation has been compared to solutions from FE computations, and agreement was obtained within time intervals relevant for solving inverse problems.

The corresponding problem for the Mindlin plate can be expected to be more complex due to the existence of several wave front speeds and lack of analytical representations of certain operators. Note, however, that the major difficulties in the case of the inhomogeneous membrane were due to the the varying wave speed resulting from the type of inhomogeneity. A thickness variation in the Mindlin plate, for instance, would not change the wave front speeds. Note also, that the difficulties of having several wave front speeds have successfully been handled for the case of Timoshenko beams [2-4,7]. 


\section{Acknowledgement}

This work has been supported by the Swedish Research Council for Engineering Sciences (TFR) and the Swedish Research Council (VR). This is gratefully acknowledged.

\section{A Operators}

The explicit representation of the operators $\mathcal{A}_{i}$ are

$$
\begin{aligned}
& \mathcal{A}_{1}=\frac{c_{2}}{2 c_{1} \tau}\left(\frac{c_{1}^{2}}{c_{2}^{2}}+\frac{2}{\cdot} \mathrm{I}_{2}(\cdot / \tau) *\right) \sin \left(c_{1} \cdot / r_{0}\right) * \\
& \mathcal{A}_{2}=\left(\frac{c_{2}^{2}}{c_{1}^{2}}-1\right)-\frac{c_{2}}{2 c_{1} \tau}\left(\frac{c_{2}^{2}}{c_{1}^{2}}+\frac{2}{\cdot} \mathrm{I}_{2}(\cdot / \tau) *\right) \sin \left(c_{1} \cdot / r_{0}\right) * .
\end{aligned}
$$

The operators $\mathcal{N}_{i}$ can be given explicitly using the operators

$$
\begin{aligned}
\mathcal{C}_{1} & =C_{1}(\cdot) *, & \mathcal{C}_{2} & =\mathcal{C}_{1}\left(1-\mathcal{C}_{1}\right)^{-1}=C_{2}(\cdot) *, \\
C_{1}(t) & =\frac{1}{2 \tau} \int_{0}^{t / \tau} \frac{\mathrm{I}_{1}(\xi) \mathrm{d} \xi}{\xi}, & C_{2}(t) & =\frac{1}{2 \tau} \mathrm{I}_{1}(t / \tau) .
\end{aligned}
$$

Then, with $q=\left(c_{2}^{2}+c_{1}^{2}\right) /\left(c_{2}^{2}-c_{1}^{2}\right)>1$, the $\mathcal{N}_{i}$ are

$$
\begin{array}{rlrl}
\mathcal{N}_{1} & =-\mathcal{C}_{2}, & \mathcal{N}_{2} & =\frac{q-1}{2}+q \mathcal{C}_{2}, \\
\mathcal{N}_{3}=\frac{q-1}{q+1} \mathcal{C}_{2}+\frac{2}{q+1} \mathcal{C}_{1} \mathcal{C}_{2}, & \mathcal{N}_{4}=c_{2}^{2} \frac{q-1}{2} \partial_{t}^{-2}\left(1+2 \mathcal{C}_{2}\right) .
\end{array}
$$

The operators $\lambda_{i}^{-1}$ are

$$
\begin{aligned}
\lambda_{1}^{-1} & =c_{1} Q_{1} *+F_{2} * Q_{2} *, & \lambda_{2}^{-1} & =c_{2} Q_{1} *+F_{1} * Q_{2} *, \\
Q_{1}(t) & =\mathrm{J}_{0}\left(c_{1} t / r_{0}\right), & Q_{2}(t) & =c_{1} c_{2} \partial_{t}^{-1} Q_{1}(t) .
\end{aligned}
$$

The $\mathcal{M}_{i}$ are given by $\mathcal{M}_{i}=M_{i}(\cdot) *$, where

$$
\begin{aligned}
M_{1}(t)=\frac{c_{1}^{2}}{c_{2}^{2} r_{0}^{2}}, \quad M_{2}(t) & =\frac{\kappa^{2} c_{1}}{r_{0}^{3}}, \quad M_{3}(t)=\frac{c_{1}}{r_{0}} \mathrm{H}(t) \sin \left(c_{1} t / r_{0}\right), \\
M_{4}(t) & =\frac{r_{0}}{2 c_{2} \kappa^{2}} \int_{0}^{t / \tau} \mathrm{I}_{0}(\xi) \mathrm{d} \xi .
\end{aligned}
$$

The multiplication by $s^{ \pm 1 / 2}$ to the wave splitting transformation kernels $\widetilde{\mathcal{B}}_{0, i j}$ and $\widetilde{\mathcal{B}}_{0, i j}^{-1}$ is equivalent to a $\mp 1 / 2$ order time integral [24]. After some simplifications, the transform kernels $B_{i j}$, where $\mathcal{B}_{i j}=B_{i j} *$, are given for the case 
$m=0$ by

$$
\begin{aligned}
& B_{11}=\left\{\begin{array}{ll}
\sqrt{\frac{\gamma_{0}}{4 \pi}} \delta(t)+\frac{\sqrt{\gamma_{0}}}{2 \pi^{3 / 2} t}\left(\frac{1}{\left(1-\gamma_{0} / t\right)} \mathrm{E}\left(t / \gamma_{0}\right)+\left(\mathrm{E}\left(t / \gamma_{0}\right)-\mathrm{K}\left(t / \gamma_{0}\right)\right)\right), & t<\gamma_{0}, \\
\frac{1}{2 \pi^{3 / 2} \sqrt{t}}\left(\frac{1}{\left(1-t / \gamma_{0}\right)} \mathrm{E}\left(\gamma_{0} / t\right)+2\left(\mathrm{E}\left(\gamma_{0} / t\right)-\mathrm{K}\left(\gamma_{0} / t\right)\right)\right), & t>\gamma_{0},
\end{array} B_{12}= \begin{cases}-\frac{c \sqrt{\gamma_{0}}}{\pi^{3 / 2}} \mathrm{~K}\left(t / \gamma_{0}\right), & t<\gamma_{0}, \\
-\frac{c \gamma_{0}}{\pi^{3 / 2} \sqrt{t}} \mathrm{~K}\left(\gamma_{0} / t\right), & t>\gamma_{0},\end{cases} \right. \\
& B_{21}=\sqrt{\frac{\pi \gamma_{0}}{4} \delta(t)+\frac{1}{\sqrt{\pi\left(t+\gamma_{0}\right)}}\left(\mathrm{E}\left(t /\left(t+\gamma_{0}\right)\right)+\frac{\gamma_{0}}{2 t}\left(\mathrm{E}\left(t /\left(t+\gamma_{0}\right)\right)-\mathrm{K}\left(t /\left(t+\gamma_{0}\right)\right)\right)\right),} \\
& B_{22}=\frac{c \gamma_{0}}{\sqrt{\pi\left(t+\gamma_{0}\right)} \mathrm{E}\left(t /\left(t+\gamma_{0}\right)\right),} \\
& B_{11}^{-1}=\sqrt{\frac{\pi}{\gamma_{0}} \delta(t)-\frac{1}{\pi^{1 / 2} t \sqrt{t+\gamma_{0}}}\left(\mathrm{~K}\left(t /\left(t+\gamma_{0}\right)\right)-\mathrm{E}\left(t /\left(t+\gamma_{0}\right)\right)\right),} \\
& B_{12}^{-1}= \begin{cases}\sqrt{\frac{1}{\pi \gamma_{0}}} \delta(t)-\frac{1}{\pi^{3 / 2} \sqrt{\gamma_{0}}\left(t-\gamma_{0}\right)}\left(\frac{\gamma_{0}}{t}\left(\mathrm{E}\left(t / \gamma_{0}\right)-\mathrm{K}\left(t / \gamma_{0}\right)\right)+\mathrm{K}\left(t / \gamma_{0}\right)\right), & t<\gamma_{0}, \\
\frac{-1}{\pi^{3 / 2} \sqrt{t}\left(t-\gamma_{0}\right)} \mathrm{E}\left(\gamma_{0} / t\right), & t>\gamma_{0} .\end{cases}
\end{aligned}
$$

The remaining two elements, $B_{21}^{-1}$ and $B_{22}^{-1}$ of the inverse transformation have not been explicitly written, but due to the re-normalization, they are essentially obtained by differentiating $B_{21}$ and $B_{11}$ with respect to $t$, respectively.

\section{B Inverse Laplace Transform by use of Asymptotics}

The time domain behaviour of the wave splitting transformation kernels, discussed at the end of Section 4, can be be obtained approximately for small values of the time variable by studying the asymptotics for large values of the Laplace variable. The starting point is the uniform asymptotic expressions for large arguments for the modified Bessel functions $\mathrm{I}_{m}[25]$

$$
\mathrm{I}_{m}\left(\tilde{\lambda}_{k} r\right) \mathrm{e}^{-s r / c_{k}} \sim \frac{\mathrm{e}^{r\left(\tilde{\lambda}_{k}-s / c_{k}\right)}}{\sqrt{2 \pi \tilde{\lambda}_{k} r}} \sum_{j=0}^{\infty} \frac{(-1)^{j} A_{j, m}}{\tilde{\lambda}_{k}^{j} r^{j}}-\mathrm{ie}^{-m \pi \mathrm{i}} \frac{\mathrm{e}^{-r\left(\tilde{\lambda}_{k}+s / c_{k}\right)}}{\sqrt{2 \pi \tilde{\lambda}_{k} r}} \sum_{j=0}^{\infty} \frac{A_{j, m}}{\tilde{\lambda}_{k}^{j} r^{j}},
$$

for $-\pi \leq \arg \tilde{\lambda}_{k} \leq 0, k=1,2,3$. For $0 \leq \arg \tilde{\lambda}_{k} \leq \pi$, one uses the connection formula $\mathrm{I}_{m}\left(\tilde{\lambda}_{k} r\right)=e^{m \pi \mathrm{i}} \mathrm{I}_{m}\left(e^{-\pi \mathrm{i}} \tilde{\lambda}_{k} r\right)$.

The $\tilde{\lambda}_{k}$ are replaced by their asymptotic expansions for large $s$. (72) may then be written as an asymptotic series in inverse powers of $s$. Each term is then transformed back to the time domain analytically, giving a series representation in the time domain for small values of the time variable. To know how to close the contours, the analyticity properties of the $\tilde{\lambda}_{k}$ must be investigated. 
The analyticity of the $\tilde{\lambda}_{l}, l=1,2$ for the Timoshenko beam, which are essentially the same, has been studied before by several authors, among them [26]. They have however presented sets of branch cuts that are not suitable for our purposes since we may expect to integrate also in the left half plane. In the Laplace domain the $\tilde{\lambda}_{k}$ are given by

$$
\tilde{\lambda}_{l}=\frac{\left(q s^{2} \pm s\left(s^{2}-\tau^{-2}\right)^{1 / 2}\right)^{1 / 2}}{c_{2}(q-1)^{1 / 2}}, \quad \tilde{\lambda}_{3}=\frac{\left(s^{2}+c_{1}^{2} / r_{0}^{2}\right)^{1 / 2}}{c_{3}}
$$

where $q=\left(c_{2}^{2}+c_{1}^{2}\right) /\left(c_{2}^{2}-c_{1}^{2}\right)>1$. Consider first $\tilde{\lambda}_{3}$. It has the branch points $s= \pm \mathrm{i} c_{1} / r_{0} \cdot \bar{\lambda}_{3}$ is analytic for $|s|>c_{1} / r_{0}$ and the branch cut may be chosen in such a way that $c_{3} \tilde{\lambda}_{3} / s \rightarrow 1$ for $s \rightarrow \infty$. Similarly, the inner square root of $\tilde{\lambda}_{l}$ is analytic for $|s|>\tau^{-1}$ and the branch cut may be chosen so that $\left(s^{2}-\tau^{-2}\right)^{1 / 2} / s \rightarrow 1$ for $s \rightarrow \infty$. Then, $\tilde{\lambda}_{l}$ are analytic for $|s|>\tau^{-1} \max \left(1,1 / \sqrt{q^{2}-1}\right)$ and the cuts may be chosen so that $c_{l} \tilde{\lambda}_{l} / s \rightarrow 1$, when $s \rightarrow \infty$. For $\tilde{\lambda}_{k}^{1 / 2}$, the principal branch is used.

Noting that the leading terms of $\tilde{\lambda}_{l}$ are $s / c_{l}$, the leading terms of the exponentials of the exponential functions in (72) for large $s$ are, for the first factor, at most a constant, and for the second factor, at most a constant after extraction of a factor $\mathrm{e}^{-2 s r / c_{k}}$. These factors may then too be expanded in negative powers of $s$. Combining the formulas (72) for the two half planes and using the asymptotic expansions of the $\tilde{\lambda}_{k}$, a power series, valid for $s \in \mathcal{U}=\left\{s:|s|>\tau^{-1} \max \left(1,1 / \sqrt{q^{2}-1}, \tau c_{1} / r\right)\right\}$, is obtained as

$$
\begin{aligned}
\mathrm{I}_{m}\left(\tilde{\lambda}_{k} r\right) \mathrm{e}^{-s r / c_{k}} & \sim \sum_{j=0}^{\infty} h_{1 k j m} s^{-(j+1 / 2)} \\
& +(-\mathrm{i}) \mathrm{e}^{-m \pi \mathrm{i}} \mathrm{e}^{-2 s r / c_{k}} \operatorname{sign}(\operatorname{Im}(s)) \sum_{j=0}^{\infty} h_{2 k j m} s^{-(j+1 / 2)}
\end{aligned}
$$

where the $h_{1 k j m}$ and the $h_{1 k j m}$ are coefficients, not specified here. The exponential factor $\mathrm{e}^{-2 s r / c_{k}}$ will just give a time shift by $2 r / c_{k}$ in the time domain so the first sum will give an expansion for values of $t$ near 0 while the second sum gives an expansion for values of $t$ near $2 r / c_{k}$. The first sum is analytic in $s \in \mathcal{U} \cap\{s: \operatorname{Re} s>0\}$. The terms in the first sum may then formally be integrated along a path to the right of the imaginary axis. Similarly, the second sum is analytic in $s \in \mathcal{U} \cap\{s: \operatorname{Re} s<0\}$. The powers of the second sum may then formally be integrated along a path to the left of the imaginary axis. By a change in variable according to $s=\omega \mathrm{e}^{-\mathrm{i} \pi / 2}$ the corresponding Fourier transforms are obtained and are identified as the distributions $(\omega \pm \mathrm{i} 0)^{-(j+1 / 2)}$, which inverse Fourier transforms are given by [27]. So, by means of the formulas

$$
\begin{aligned}
\mathcal{L}^{-1}\left[s^{-(j+1 / 2)}\right] & =t_{+}^{j-1 / 2} / \Gamma(j+1 / 2), \\
\mathcal{L}^{-1}\left[\operatorname{sign}(\operatorname{Im}(s)) s^{-(j+1 / 2)}\right] & =\mathrm{i}(-1)^{j} t_{-}^{j-1 / 2} / \Gamma(j+1 / 2),
\end{aligned}
$$


expansions for $t$ near 0 and $2 r / c_{k}$ respectively, are found as

$$
\sum_{j=0}^{\infty} \frac{h_{1 k j m}}{\Gamma(j+1 / 2)} t_{+}^{j-1 / 2}, \quad \mathrm{e}^{-m \pi \mathrm{i}} \sum_{j=0}^{\infty} \frac{(-1)^{j} h_{2 k j m}}{\Gamma(j+1 / 2)}\left(t-2 r / c_{k}\right)_{-}^{j-1 / 2} .
$$

It turns out, though we do not prove it, that $h_{2 k j m}=(-1)^{j} h_{1 k j m}$. Then, $\mathcal{L}^{-1}\left[\mathrm{I}_{m}\left(\tilde{\lambda}_{k} r\right) \mathrm{e}^{-s r / c_{k}}\right]$ is an even function about $t=r / c_{k}$ for even $m$ and an odd function for odd $m$, which simplifies the computation of the functions in the time domain somewhat.

The time domain representation of $\mathrm{K}_{m}\left(\tilde{\lambda}_{k} r\right) \mathrm{e}^{s r / c_{k}}$ for small $t$ may be computed in an analogous way. The asymptotic expression for large arguments is

$$
\mathrm{K}_{m}\left(\tilde{\lambda}_{k} r\right) \mathrm{e}^{s r / c_{k}} \sim \frac{\mathrm{e}^{-z_{k}+s r / c_{k}}}{\sqrt{2 \pi \tilde{\lambda}_{k} r}} \sum_{j=0}^{\infty} \frac{A_{j, m}}{\tilde{\lambda}_{k}^{j} r^{j}} .
$$

From the analysis for the $\tilde{\lambda}_{k}$ it is seen this series is analytic for $s \in \mathcal{U} \cap\{s$ : $\operatorname{Re} s>0\}$. Expansion into powers of $s$ and use of (74) gives

$$
\sum_{j=0}^{\infty} \frac{h_{3 i j m}}{\Gamma(j+1 / 2)} t_{+}^{(j-1 / 2)}
$$

where $h_{3 i j m}$ are coefficients similar to $h_{1 i j m}, h_{2 i j m}$.

\section{Discretization of singularities in the numerical computation}

The convolution of power singularities with smooth functions are interpreted as CPV integrals. In (63), some kernels contain power singularities, which propagate along known characteristics. When the computational region is discretised, the characteristic lines will pass through discrete points, as well as between them. We thus want to be able to treat both cases within the frame of the trapezoidal rule. The numerical implementation of these integrals has been done by modifying the discretization of the singular function in the neighbourhood of the singular point.

Consider the singular function $g(t)=\left(t-t^{\prime}\right)^{-1}$. At a certain discrete point in space, $t^{\prime}=k h \pm \delta$, where $h$ is the time step, $k$ is an integer and $0 \leq \delta \leq h / 2$. Then, $g(t)$ has been approximated by

$$
g_{\mathrm{app}}(t)=(t-k h \pm \delta)^{-1} \approx w_{1}(t-k h)^{-1}+w_{2}(t-k h \pm h / 2)^{-1},
$$

where $w_{1}=(h-2 \delta) / h$ and $w_{2}=2 \delta / h$. The absolute error is then large for $t \approx k h \pm \delta$, but the interated error is small. When $g_{\text {app }}(t)$ is convolved with some smooth function $f$, use of the CPV definition, Taylor expansion and the 
trapezoidal rule will give the result $I$, say. The modified function $g_{\mathrm{app}}^{\bmod }$ is then defined as the discrete function that give the result $I$, when convolved with $f$, using only the trapezoidal rule and the same discretization. $g_{\mathrm{app}}^{\mathrm{mod}}$ is then given by

$$
\begin{aligned}
g_{\mathrm{app}}^{\mathrm{mod}}=\left[g(0), g(h), \ldots, g((k-2) h), \frac{-w_{1}}{2 h}+\frac{-w_{2}}{h(1 \mp 1 / 2)},\right. \\
\left.\frac{2 w_{2}}{ \pm h}, \frac{w_{1}}{2 h}+\frac{w_{2}}{h(1 \pm 1 / 2)}, g((k+2) h), \ldots\right]
\end{aligned}
$$

Logarithmic singularities have been represented in terms of the integral of a discretised and modified power singularity. The reason for this is to obtain a uniform representation of logarithmic singularities in the imbedding equation.

\section{References}

[1] S. He, S. Ström, V. H. Weston, Time Domain Wave-Splittings and Inverse Problems, Monographs in Electrical and Electronic Engineering, Oxford University Press, Oxford, 1998.

[2] D. V. J. Billger, P. D. Folkow, The imbedding equations for the Timoshenko beam, Journal of Sound and Vibration 209 (4) (1998) 609-634.

[3] D. V. J. Billger, P. D. Folkow, Wave propagators for the Timoshenko beam, Wave Motion 37 (4) (2003) 313-332.

[4] D. V. J. Billger, D. J. N. Wall, A time domain algorithm for the reflection of waves on a viscoelastically restrained Timoshenko beam, Quarterly Journal of Mechanics and Applied Mathematics 52 (2) (1999) 211-236.

[5] P. D. Folkow, K. Kreider, Direct and inverse problems on nonlinear rods, Mathematics and Computers in Simulation 50 (5-6) (1999) 577-595.

[6] M. Romeo, Wave splitting in linear viscoelasticity, European Journal of Mechanics A-Solids 18 (1999) 539-55.

[7] P. D. Folkow, Time domain inversion of a viscoelastically restrained Timoshenko beam, Inverse Problems 15 (2) (1999) 551-562.

[8] V. H. Weston, Invariant imbedding for the wave equation in three dimensions and the application to the direct and inverse problems, Inverse Problems 6 (6) (1990) 1075-1105.

[9] V. H. Weston, Invariant imbedding and wave splitting in $\mathrm{R}^{3}:$ II. the Green function approach to inverse scattering, Inverse Problems 8 (6) (1992) 919-947.

[10] A. Karlsson, G. Kristensson, Wave splitting in the time domain for a radially symmetric geometry, Wave Motion 12 (1990) 197-211. 
[11] K. L. Kreider, Time dependent direct and inverse electromagnetic scattering for the dispersive cylinder, Wave Motion 11 (1989) 427-440.

[12] K. L. Kreider, A wave splitting approach to time dependent inverse scattering for the stratified cylinder, SIAM Journal of Applied Mathematics 49 (3) (1989) 932-943.

[13] S. P. Timoshenko, On the correction for shear of the differential equation for transverse vibrations of prismatic bars, Phil. Mag. XLI (1921) 744-746, reprinted in The Collected Papers of Stephen P. Timoshenko, McGraw-Hill, London 1953.

[14] P. Olsson, G. Kristensson, Wave splitting of the Timoshenko beam equation in the time domain, Zeitschrift fur Angewandte Mathematik und Physik 45 (1994) $866-881$.

[15] P. D. Folkow, P. Olsson, G. Kristensson, Time domain Green functions for the homogeneous Timoshenko beam, Quarterly Journal of Mechanics and Applied Mathematics 51 (1) (1998) 125-141.

[16] R. J. Krueger, R. L. Ochs, A Green's function approach to the determination of internal fields, Wave Motion 11 (1989) 525-543.

[17] R. D. Mindlin, Influence of rotatory inertia and shear on flexural motions of isotropic, elastic plates, Journal of Applied Mechanics 18 (1951) 31-38.

[18] C. Vemula, A. N. Norris, Flexural wave propagation and scattering on thin plates using Mindlin theory, Wave Motion 26 (1997) 1-12.

[19] A. Erdélyi, Tables of Integral Transforms, Vol. 1 of The Bateman Manuscript Project, McGraw-Hill, New York, 1954.

[20] F. Oberhettinger, L. Badii, Tables of Laplace Transforms, Springer-Verlag, Berlin, 1973.

[21] P. D. Folkow, Wave propagation in structural elements - direct and inverse problems in the time domain, Ph.D. thesis, Chalmers University of Technology (1998).

[22] D. S. Jones, Infinite integrals and convolutions, Proceedings of the Royal Society of London A 371 (1980) 479-508.

[23] A. Boström, Time-dependent scattering by a bounded obstacle in three dimensions, Journal of Mathematical Physics 23 (8) (1982) 1444-1450.

[24] K. B. Oldham, J. Spanier, The Fractional Calculus, Vol. 111 of Mathematics in science and engineering, Academic Press, London, 1974.

[25] C. H. Wilcox, R. E. Langer (Eds.), Asymptotic Solutions of Differential Equations and Their Applications, Publication of the Mathematics Research Center, United States Army, University of Wisconsin., Wiley, New York, 1964. 
[26] J. Miklowitz, The Theory of Elastic Waves and Waveguides, 2nd Edition, North-Holland series in applied mathematics and mechanics, North-Holland, Amsterdam, 1978.

[27] I. M. Gel'fand, G. E. Shilov, Generalized Functions, Vol. 1, Academic Press, London, 1964. 